Article

\title{
A Subcarrier-Pair Based Resource Allocation Scheme Using Proportional Fairness for Cooperative OFDM-Based Cognitive Radio Networks
}

\section{Yongtao Ma, Liuji Zhou * and Kaihua Liu}

School of Electronic Information Engineering, Tianjin University, 92 Weijin Road, Nankai District, Tianjin 300072, China; E-Mails: mayongtao@tju.edu.cn (Y.M.); liukaihua@tju.edu.cn (K.L.)

* Author to whom correspondence should be addressed; E-Mail: honglin929@tju.edu.cn; Tel.: +86-158-2245-5869.

Received: 28 June 2013; in revised form: 5 August 2013 / Accepted: 6 August 2013 /

Published: 9 August 2013

\begin{abstract}
The paper presents a joint subcarrier-pair based resource allocation algorithm in order to improve the efficiency and fairness of cooperative multiuser orthogonal frequency division multiplexing (MU-OFDM) cognitive radio (CR) systems. A communication model where one source node communicates with one destination node assisted by one half-duplex decode-and-forward (DF) relay is considered in the paper. An interference-limited environment is considered, with the constraint of transmitted sum-power over all channels and aggregate average interference towards multiple primary users (PUs). The proposed resource allocation algorithm is capable of maximizing both the system transmission efficiency and fairness among secondary users (SUs). Besides, the proposed algorithm can also keep the interference introduced to the PU bands below a threshold. A proportional fairness constraint is used to assure that each SU can achieve a required data rate, with quality of service guarantees. Moreover, we extend the analysis to the scenario where each cooperative SU has no channel state information (CSI) about non-adjacent links. We analyzed the throughput and fairness tradeoff in CR system. A detailed analysis of the performance of the proposed algorithm is presented with the simulation results.
\end{abstract}

Keywords: cognitive radio; cooperative communication; resource allocation; proportional fairness; spectrum sharing 


\section{Introduction}

Cognitive radio technology (CR) has been proposed as a relatively new concept for improving the overall utilization of spectrum bands. This promising technology can allow the unlicensed secondary users (SUs, also referred to as CR users or CRUs) to access those frequency bands which are not currently being used by licensed primary users (PUs) in a given geographical area [1,2]. Cooperative communication technology [3] allows network nodes with single antennas to use other network nodes' antennas to transmit data, which can generate a virtual multiple-input multiple-output (MIMO) system. Cooperative spectrum sensing is a viable sensing technique to enhance spectral utilization efficiency of secondary users while ensuring the quality of service (QoS) of primary users [4]. In a CR network, SUs are allowed to transmit over the frequency bands of PUs as long as the resulting aggregate interference is kept below a certain threshold. This threshold is known as interference temperature constraint or interference power constraint [1]. As SUs can design power and subcarrier allocation strategies subject to such interference power constraints, the interference introduced to PUs is effectively controlled. A great deal of resource allocation algorithms and interference control strategies has been proposed for spectrum-sharing CR networks. For example, the optimal power allocation strategies to maximize the transmitted data rate of the secondary user with an effective protection of the primary user were studied in [5,6] for spectrum-sharing CR networks.

Orthogonal frequency division multiplexing (OFDM) is an attractive modulation scheme for users in a CR system due to its flexibility in allocating resources among SUs. Since both SUs and PUs may exist in side-by-side bands, yet have different access technologies, mutual interference is the limiting factor for the performance of both networks. Thus, using of the classical subcarrier allocation and power loading algorithms, such as uniform power but variable rate and water-filling algorithms maximizing the transmission capacity of an OFDM-based conventional wireless network may result in higher mutual interference in the PUs' band. There is only one group of users in such a wireless network, i.e., PUs, for a CR system.

According to the latest literature on resource allocation in cooperative communication [7-17], the relay users in the system do not transmit their own data and merely help other non-relay users transmit data. In some wireless applications such as cellular networks, however, each user has its own data to transmit so that it should allocate its total constrained power and subcarriers properly in transmitting its own data and relaying other users' data [18,19]. Tourki [19] focused on efficiency issues by studying how to maximize the total transmitted data rate in non-orthogonal amplify-and-forward (AF) cooperative scheme, which ignores the fairness among the cooperative users. According to [20,21], equal power allocation (EPA) among subcarriers was proposed to separate the user selection from the power of subcarrier. With EPA, the EPA-PRG (proportional rate greedy) [22] algorithm is proposed to maximize the system throughput while keeping the fairness. However, cooperative transmission technology isn't applied in this algorithm. In [23], a linear water-filling scheme (LWF-PI) was proposed. This algorithm maximized the overall transmitted data rate of the CR system while keeping the interference introduced to the PU bands below a threshold. However, the fairness among users was ignored. Chandrashekar et al. [24] proposed an algorithm which is capable of maximizing the total transmitted data rate and achieving a high proportional fairness index. However, this algorithm cannot be applied to the CR network where we must adjust the interference introduced to the PU bands below 
a threshold. Tan [25] proposed a joint subcarrier and power algorithm based on Blotto games. This algorithm can achieve a good trade-off performance between fairness and efficiency in OFDMA-based cognitive radio network (CRN), but it cannot obtain the effectiveness of multiuser diversity for the SUs without ability to generate a virtual MIMO system.

A novel scheme was presented in [26] for the allocation of subcarriers, rates, and power in orthogonal frequency-division multiple-access (OFDMA) networks. The resource-allocation problem was solved by decomposing it into a hierarchy of sub-problems. A joint subcarrier and power allocation algorithm was presented in [27] for cooperative MU-OFDM CR systems. In [28], a survey of resource allocation and scheduling schemes in OFDMA wireless networks was presented. Nader et al. in [29] considered the practical case in which only partial CSI for the wireless channel between the secondary base station and SUs is available at the secondary base station. They formulated the resource allocation problem in the secondary network as an optimization problem in which the objective was to maximize the weighted sum rate of the secondary users. A novel sub-channel and transmission power allocation scheme was proposed in [30] for multi-cell OFDMA networks with CR functionality. Tianxiang et al. in [31] discussed optimization over the relay assignment, subcarrier allocation, per node power control, and heterogeneous quality-of-service (QoS) provisioning. Sabit et al. in [32] investigated the performance of an OFDM-based CR spectrum sharing communication system that assumed random allocation and absence of the PU channel occupation information. Hong $\mathrm{Xu}$ et al. in [33] formulated a unifying optimization framework based on Nash bargaining solutions to fairly and efficiently allocate resources between primary and secondary networks, in both decentralized and centralized settings. As the optimal resource allocation scheme was highly complex, G. B. et al. [34] proposed a low complexity suboptimal subcarrier and power allocation scheme. They also proposed a suboptimal subcarrier allocation scheme that can guarantee a certain level of fairness among CR users. Naeem et al. introduced in [35] a hybrid heuristic algorithm for the relay assignment and power allocation problem which is a non-convex mixed-integer non-linear optimization problem, and this problem is generally non-deterministic polynomial-time (NP)-hard.

In this paper, a joint subcarrier-pair based resource allocation algorithm in order to improve both efficiency and fairness index is presented first. The definition of fairness is borrowed from the networking literature. In contrast with [36], where large channel fluctuations are intentionally created with "dumb" antennas for long-term proportional fairness resource allocation, this paper proposes a subcarrier-pair based resource allocation algorithm to maintain proportional rates among SUs for each channel realization, which ensures the rates of different SUs to be proportional in any time scale of interest. By formulating the resource allocation and pairing problem in this way, it will be shown that a high transmitted data rate for all SUs (even those with poor channel gains) can be achieved with low computational complexity. Moreover, we extend the analysis to the case in which each SU can only have access to CSI of its adjacent links. This is a more realistic scenario when network nodes are mobile and the timely CSI cannot be exchanged between cooperative users. Consequently, each user can only have access to statistical CSI of non-adjacent links. It is shown that the system performance deteriorates due to limited CSI but still outperforms that of equal power allocation scheme. The key contributions of this work are: 
1. It is considered that SUs need to transmit their own data directly to the destination, and in the next phase they also help their partner forward the data received in previous phase to the destination. Simulation results show that in the same situations the system transmitted data rate by proposed algorithm is the highest than that by LWF-PI algorithm [23], EPA algorithm [21] and the Optimal Scheme [37].

2. The proposed subcarrier-pair based resource allocation algorithm ensures the rates of different SUs to be proportional in any time scale of interest, simulation results shown that a high transmitted data rate for all SUs (even those with poor channel gains) can be achieved.

3. It is considered that SU has no CSI about non-adjacent link. In this case, we take full advantage of the statistical information of the non-adjacent links.

Notation: In this paper, a variable with "underline" 므 denotes the temporary optimal values within each iteration process, the "double underline" statistical average value. $E(\boldsymbol{\square})$ denotes the expectation operator, and $\boldsymbol{\overline { \boldsymbol { m } }}$ denotes the optimal value when only partial CSI can be obtained by SUs.

\section{System Model and Problem Formulation}

We consider a hybrid network consisting of a primary network (PRN) and a cognitive radio network (CRN) as shown in Figure 1. The CRN consists of a CR access point (AP) and $2 K$ SUs. The PRN and CRN co-exist within the same geographical area. The access mechanism/modulation format in SUs' band is OFDM. Our focus is mainly on the uplink radio resource allocation in the CRN. The SUs are trying to find the opportunity to access to the AP.

Figure 1. A cooperative MU-OFDM CR uplink system.

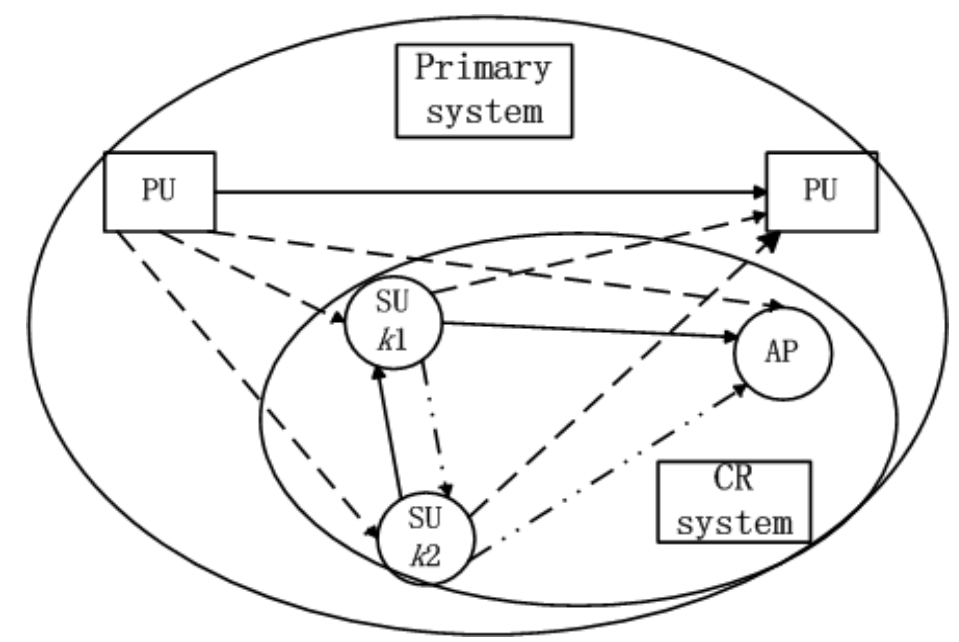

According to [37,38], we also consider that the frequency bands of bandwidth $B_{1}, B_{2}, \ldots, B_{L}$ which have been occupied by $L$ PUs are sensed by the CR system and known to SU transmitters. Every two SUs form a cooperative partner and they are relay node for each other. As shown in Figure 2, the $k$ th $(1 \leq k \leq K)$ cooperative partner consists of two SU transmitters, $k 1$ and $k 2$. As is assumed in [23,37,38], we consider the same side-by-side CR radio access model. The unoccupied bandwidth sensed by SUs 
for opportunistic spectrum access is located on each side of $L$ PU bands as shown in Figure 3. The available bandwidth for CR transmission is divided into $N$ subcarriers based on OFDM system. It is considered that the access mechanism/modulation format in PUs' band is not known to the CR system and the bandwidth for each CR subcarriers is $\Delta f H z$. Some symbols are shown in Table 1.

Figure 2. Model for cooperative transmission.

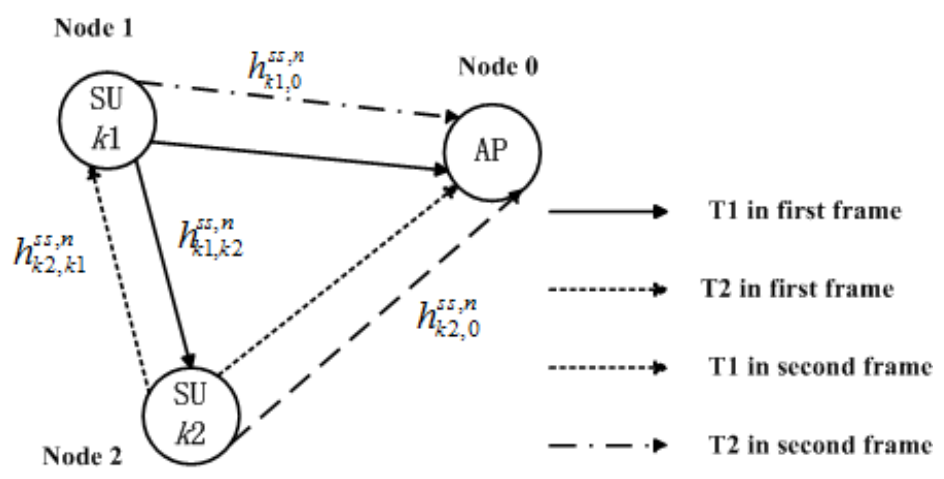

Figure 3. spectrum access model of cognitive radio system.

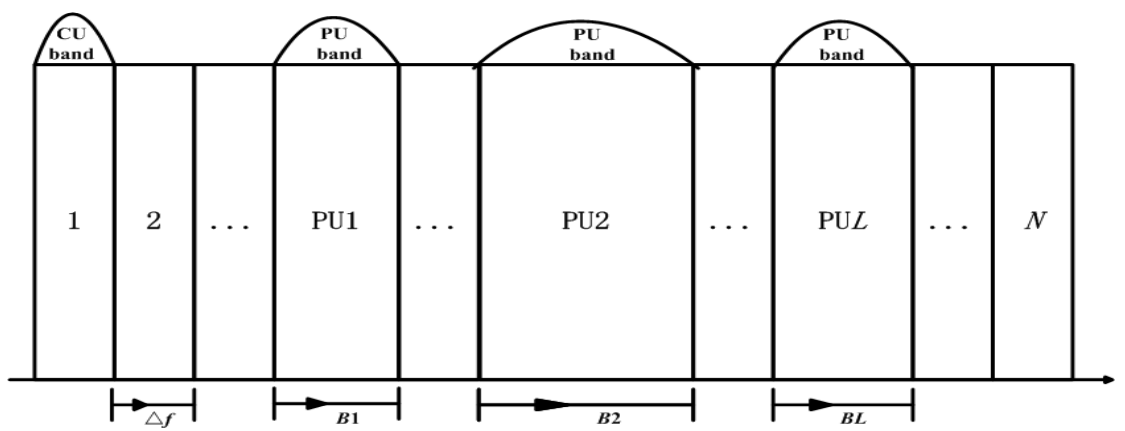

In general, there are three instantaneous fading gains in the uplink transmission scenario shown in Figure 1:

(1) The gains between the SU's transmitter and SU's receiver or AP for the $n$th subcarrier denoted as $h_{k i, k j}^{s s, n}, h_{k i, 0}^{s s, n}$, respectively.

(2) The gains between the SU's transmitter and $l$ th PU's receiver, denoted as $h_{k i, p l}^{s p, n}$.

(3) The gains between the $l$ th PU's transmitter and the SU's receiver or AP, denoted as $h_{p l, k i}^{p s, n}, h_{p l, 0}^{p s, n}$, respectively.

The channel gains are modeled as independent zero-mean complex Gaussian random variables, where $k i$ denotes the $i$ th $\mathrm{SU}$ in $k$ th cooperation partner and $p l$ denotes the $l$ th PU band. According to [39], it is considered that these instantaneous fading gains are perfectly known at the SU's transmitter. Specifically, we assume that the SU's receiver can estimate channel gains $h_{k i, k j}^{s s, n}$ and $h_{p l, k i}^{p s, n}$ and report to the CR transmitter. In Section 3.2, we will study the case where the instantaneous fading gains of the non-adjacent links are not perfectly known at the SU transmitter but the statistical information of the non-adjacent links are known at the SU transmitter. Moreover, it is assumed that primary receiver can estimate the channel $h_{k i, p l}^{s p, n}$ which is reported to the SU transmitter through a common control channel. 
Table 1. Table of Symbols.

\begin{tabular}{|c|c|}
\hline Symbol & Definition \\
\hline$K$ & Number of cooperative partners in the CR network \\
\hline$N$ & Number of subcarriers \\
\hline$\Delta f$ & Bandwidth of a subcarrier \\
\hline$T_{S}$ & Length of a slot \\
\hline$P_{k 1,1}^{n, \mathrm{SP}(n)}$ & Transmission power of $k 1^{\text {th }} \mathrm{SU}$ on subcarrier $n$ in the first frame \\
\hline$P_{k 1,2}^{n, \operatorname{SP}(n)}$ & Transmission power of $k 1^{\text {th }} \mathrm{SU}$ on subcarrier $n$ in the second frame \\
\hline$P_{k 2,1}^{n, \operatorname{SP} 1(n)}$ & Transmission power of $k 2^{\text {th }} \mathrm{SU}$ on subcarrier $n$ in the first frame \\
\hline$P_{k 2,2}^{n, \mathrm{SP} 2(n)}$ & Transmission power of $k 2^{\text {th }} \mathrm{SU}$ on subcarrier $n$ in the second frame \\
\hline$h_{k i, k j}^{s s, n}(i \neq j)$ & $\begin{array}{l}\text { the channel gain of the communication link from the kith } \mathrm{SU} \text { to the } k j \text { th } \mathrm{SU} \\
\text { user on the } n \text {th subcarrier }\end{array}$ \\
\hline$h_{k i, 0}^{s s, n}$ & $\begin{array}{l}\text { the channel gain of the communication link } \\
\text { from the kith SU to AP on the } n \text {th subcarrier }\end{array}$ \\
\hline$h_{k i, p l}^{s p l, n}$ & $\begin{array}{l}\text { the channel gain of the interference link } \\
\text { from } l \text { th PU to kith SU user receiver on the } n \text {th subcarrier }\end{array}$ \\
\hline$h_{p l, k i}^{p s, n}$ & $\begin{array}{l}\text { the channel gain of the interference link } \\
\text { from } l \text { th PU to kith SU user receiver on the } n \text {th subcarrier }\end{array}$ \\
\hline$h_{p l, 0}^{p s, n}$ & $\begin{array}{l}\text { the channel gain of the interference link } \\
\text { from } l \text { th PU to AP receiver on the } n \text {th subcarrier }\end{array}$ \\
\hline$\left\{z_{k 1}^{(i)}, z_{k 2}^{(i)}, z_{0}^{(i)}, i=1,2,3,4\right\}$ & the additive noises at the corresponding node \\
\hline$\left\{\vartheta_{k 1}^{(i)}, \vartheta_{k 2}^{(i)}, \vartheta_{0}^{(i)}, i=1,2,3,4\right\}$ & the interference introduced by the PUs into corresponding node \\
\hline
\end{tabular}

\subsection{Cooperative Transmission among SUs}

The scenario of a three-node DF diversity model is considered, where one source communicates with one destination assisted by one half-duplex relay, as shown in Figure 2. One transmission period is divided into two consecutive frames. Communication takes place in two phases (listening phase T1 and relaying phase $\mathrm{T} 2$, the definition is according to the working state of relay user) for each frame. The power allocation scheme for $k$ th cooperative partner on subcarrier $n$ is shown in Table 2. The source node broadcasts its signal to relay and AP in T1, whereas the relay and AP listen. The relay decodes the signal and forwards it to AP in T2. It is denoted that the subcarrier $n$ in T1 is pairing with subcarrier SP1 $(n)$ in T2 for first frame, and pairing with subcarrier SP2 $(n)$ for second frame. In the first frame, $k 2$ th $\mathrm{SU}$ receives data in this time slot while $k 1$ th $\mathrm{SU}$ transmits a symbol $x_{k 1}(t)$ with power level $P_{k 1,1}^{n, \mathrm{SP}(n)}$ on $n$th subcarrier in T1. The symbol is received by node 0 (AP) and overheard by $k 2$ th SU as:

$$
\begin{aligned}
& y_{k 1,0}(t)=h_{k 1,0}^{s s, n} \sqrt{P_{k 1,1}^{n, \operatorname{SP} 1(n)}} x_{k 1}(t)+z_{0}^{(1)}(t)+\vartheta_{0}^{(1)}(t) \\
& y_{k 1, k 2}(t)=h_{k 1, k 2}^{s s, n} \sqrt{P_{k 1,1}^{n, \operatorname{SP1}(n)}} x_{k 1}(t)+z_{k 2}^{(1)}(t)+\vartheta_{k 2}^{(1)}(t)
\end{aligned}
$$


Table 2. Power allocation scheme for $k$ th cooperative partner on subcarrier $n$.

\begin{tabular}{lcccc}
\hline & T1 in First Frame & T2 in First Frame & T1 in Second Frame & T2 in Second Frame \\
\hline $\mathrm{SU}_{k 1}$ & $P_{k 1,1}^{n, \mathrm{SP} 1(n)}$ & 0 & 0 & $P_{k 1,2}^{n, \mathrm{SP} 2(n)}$ \\
$\mathrm{SU}_{k 2}$ & 0 & $P_{k 2,1}^{n, \mathrm{SP} 1(n)}$ & $P_{k 2,2}^{n, \mathrm{SP}(n)}$ & 0 \\
\hline
\end{tabular}

During this interval, the $k 2$ th SU decodes its overheard signal as $x_{k 1}^{*}(t)$ and transmits it to the AP on $\operatorname{SP} 1(n)$ subcarrier in $\mathrm{T} 2$ with the power level $P_{k 2,1}^{n, \operatorname{SP} 1(n)}$. Then the AP receives the signal as:

$$
y_{k 2,0}(t)=h_{k 2,0}^{s s, \operatorname{SP} 1(n)} \sqrt{P_{k 2,1}^{n, \operatorname{SP} 1(n)}} x_{k 1}^{*}(t)+z_{0}^{(2)}(t)+\vartheta_{0}^{(2)}(t)
$$

In the second frame, the roles of $k 1$ th SU and $k 2$ th SU are reversed. Similarly, $k 2$ th SU transmits a symbol $x_{k 2}(t)$ with power level $P_{k 2,2}^{n, \mathrm{SP} 2(n)}$ on the nth subcarrier in T1. The symbol is received by node 0 (AP) and overheard by $k 1$ th $\mathrm{SU}$ as:

$$
\begin{aligned}
& y_{k 2,0}(t)=h_{k 2,0}^{s s, n} \sqrt{P_{k 2,2}^{n, \mathrm{SP} 2(n)}} x_{k 2}(t)+z_{0}^{(3)}(t)+\vartheta_{0}^{(3)}(t) \\
& y_{k 2, k 1}(t)=h_{k 2, k 1}^{s s, n} \sqrt{P_{k 2,2}^{n, \mathrm{SP} 2(n)}} x_{k 2}(t)+z_{k 1}^{(3)}(t)+\vartheta_{k 1}^{(3)}(t)
\end{aligned}
$$

In $\mathrm{T} 2$ of second frame, the AP node receives the noisy signal which is relayed by $k 1$ th $\mathrm{SU}$ with the power level $P_{k 1,2}^{n, \mathrm{SP} 2(n)}$, i.e.:

$$
y_{k 1,0}(t)=h_{k 1,0}^{s s, \mathrm{SP} 2(n)} \sqrt{P_{k 1,2}^{n, \mathrm{SP} 2(n)}} x_{k 2}^{*}(t)+z_{0}^{(4)}(t)+\vartheta_{0}^{(4)}(t)
$$

\subsection{Mutual Interference between PU Bands and CR Users}

In the MU-OFDM CR system, due to the coexistence of PUs and SUs in side by side bands, it is necessary to consider the mutual interference between PUs and SUs. There are two types of interference in the system. One is introduced by the PUs into the SUs band, and the other is introduced by the SUs into the PUs' band. In what follows, we provide brief description and mathematical models for interference between SUs and PUs.

\subsubsection{The Interference Introduced into PUs by SUs}

$\mathrm{CR}$ interference is introduced into the PU spectrum by $\mathrm{CR}$ out-of-band (OOB) emissions. OOB emissions arise as a result of transmit pulse shaping such that a portion of the CR radiated power in a vacant subcarrier is leaked into neighboring bands occupied by the PUs. According to [23], the interference factor which is the integration of the power density spectrum of the $n$th subcarrier across the $l$ th PU band, and can be written as:

$$
S_{k i, p l}^{n}\left(d_{n l}\right)=\left|h_{k i, p l}^{s p, n}\right|^{2} T s \int_{d_{n l}-\frac{B_{l}}{2}}^{d_{n l}+\frac{B_{l}}{2}}\left(\frac{\sin (\pi f T s)}{\pi f T s}\right)^{2} d f
$$

where $T s$ denotes the symbol duration, $d_{n l}$ denotes the distance in frequency between the $n$th subcarriers of SU band and $l$ th PU band, and $B_{l}$ represents occupied bandwidth by $l$ th PU. It can be 
shown from Equation (5) that the interference to PU band is related to the distance between SU band and PU band.

\subsubsection{The Interference Introduced into SUs by PUs}

The interference introduced into kith SU and AP node transmitting in $n$th subcarrier by $l$ th PU can be denoted as $J_{p l, k i}^{n}, J_{p l, 0}^{n}$, respectively. According to [37], the interference value $J_{p l, k i}^{n}, J_{p l, 0}^{n}$ can be written as:

$$
\begin{aligned}
& J_{p l, k i}^{n}\left(d_{n l}, P_{P U}\right)=\left|h_{p l, k i}^{s p, n}\right|^{2} \int_{d_{n l}-\frac{\Delta f}{2}}^{d_{n l}} E\left\{\frac{\Delta f}{2} E\left\{I_{N}\left(w, \phi_{P U}\left(e^{j w}\right)\right)\right\} d w\right. \\
& J_{p l, 0}^{n}\left(d_{n l}, P_{P U}\right)=\left|h_{p l, 0}^{s p, n}\right|^{2} \int_{d_{n l}-\frac{\Delta f}{2}}^{d_{n l}+\frac{\Delta f}{2}} E\left\{I_{N}\left(w, \phi_{P U}\left(e^{j w}\right)\right)\right\} d w \\
& E\left\{I_{N}\left(w, \phi_{P U}\left(e^{j w}\right)\right\}=\frac{1}{2 \pi M} \int_{-\pi}^{\pi} \phi_{P U}\left(e^{j w}\right)\left(\frac{\sin (w-\varsigma) M / 2}{\sin (w-\varsigma) / 2}\right)^{2} d \varsigma\right.
\end{aligned}
$$

where $w$ represents the frequency normalized to the sampling frequency, $E\left\{I_{N}(\bullet)\right\}$ is the power density spectrum of the PU signal after $M$-fast Fourier transform (FFT) processing, $\phi_{P U}\left(e^{j w}\right)$ is the power density spectrum of the PU signal. The PU signal has been taken to be an elliptically filtered white noise process with amplitude $P_{P U}$.

According to [40], using a relay is advantageous when:

$$
\begin{aligned}
& \text { frame 1: } \min \left(\frac{\left|h_{k 1, k 2}^{s s, n}\right|^{2}}{\sigma^{2}+\sum_{l=1}^{L} J_{p l, k 2}^{n}}, \frac{\left|h_{k 2,0}^{s s, \mathrm{SP} 1(n)}\right|^{2}}{\sigma^{2}+\sum_{l=1}^{L} J_{p l, 0}^{\mathrm{SP} 1(n)}}\right) \geq \frac{\left|h_{k 1,0}^{s s, n}\right|^{2}}{\sigma^{2}+\sum_{l=1}^{L} J_{p l, 0}^{n}} \\
& \text { frame 2: } \min \left(\frac{\left|h_{k 2, k 1}^{s s, n}\right|^{2}}{\sigma^{2}+\sum_{l=1}^{L} J_{p l, k 1}^{n}}, \frac{\left|h_{k 1,0}^{s s, \mathrm{SP} 2(n)}\right|^{2}}{\sigma^{2}+\sum_{l=1}^{L} J_{p l, 0}^{\mathrm{SP}(n)}}\right) \geq \frac{\left|h_{k 2,0}^{s s, n}\right|^{2}}{\sigma^{2}+\sum_{l=1}^{L} J_{p l, 0}^{n}}
\end{aligned}
$$

in selective DF mode. It is considered that the link of source node $\rightarrow$ relay node and link of relay node $\rightarrow$ destination node are better than that of source node $\rightarrow$ destination node. Otherwise, the relay keeps idle on subcarrier $n$ in the relaying phase for $x_{k 2}^{*}(t)$ or $x_{k 1}^{*}(t)$. In this paper, we just consider the case in which the relays keep working on each subcarrier, i.e., the Equation (7) is always true.

\section{Optimization Problem Formulation}

In this section, we analyze the joint optimization of subcarrier-pair based resource allocation algorithm for OFDM-DF based on full CSI and partial CSI, respectively. We are interested in how each SU allocates its power properly across its own data and its relayed data so as to maximize the system transmitted data rate while maintaining reasonable fairness between SUs. The optimization problem is formulated firstly and then solved in the dual domain. It is assumed that the PUs have a constant-rate, constant-power transmission, while the SUs are capable to adjust transmit power over different fading states based on the CSI of the CR network. We study a type of constraint imposed over the secondary transmission to protect the PUs by limiting the interference introduced to the PUs below a threshold. 


\subsection{Resource Allocation and Subcarrier Pairing Scheme Based on the OFDM-DF}

The CR AP combines the received signals from the source node in T1 and the relay node in T2 through the maximal ratio combining. The transmit power is adjusted in each SU's transmitter. According to [27] and [41], when the link of source node->relay node transmission is successful for entire DF process, the transmission rate of $k 1$ th $\mathrm{SU}$ and $k 2$ th $\mathrm{SU}$ at $n$ subcarrier in relaying mode, which is connected via the Shannon capacity formula, can be shown as $I_{k 1}^{n, \operatorname{SP} 1(n)}$ and $I_{k 2}^{n, \operatorname{SP2}(n)}$, respectively:

$$
\begin{aligned}
& I_{k 1}^{n, \mathrm{SP} 1(n)}=\frac{\Delta f}{4} \log _{2}\left\{1+\min \left(\left|h_{k 1, k 2}^{s s, n}\right|^{2} \frac{P_{k 1,1}^{n, \mathrm{SP} 1(n)}}{\sigma^{2}+\sum_{l=1}^{L} J_{p l, k 2}^{n}}\left|h_{k 1,0}^{s s, n}\right|^{2} \frac{P_{k 1,1}^{n, \mathrm{SP} 1(n)}}{\sigma^{2}+\sum_{l=1}^{L} J_{p l, 0}^{n}}+\left|h_{k 2,0}^{s s, \operatorname{SP} 1(n)}\right|^{2} \frac{P_{k 2,1}^{n, \mathrm{SP} 1(n)}}{\sigma^{2}+\sum_{l=1}^{L} J_{p l, 0}^{\mathrm{SP} 1(n)}}\right)\right\} \\
& I_{k 2}^{n, \mathrm{SP} 2(n)}=\frac{\Delta f}{4} \log _{2}\left\{1+\min \left(\left|h_{k 2, k 1}^{s s, n}\right|^{2} \frac{P_{k 2,2}^{n, \mathrm{SP} 2(n)}}{\sigma^{2}+\sum_{l=1}^{L} J_{p l, k 1}^{n}},\left|h_{k 2,0}^{s s, n}\right|^{2} \frac{P_{k 2,2}^{n, \mathrm{SP} 2(n)}}{\sigma^{2}+\sum_{l=1}^{L} J_{p l, 0}^{n}}+\left|h_{k 1,0}^{s, \mathrm{SP} 2(n)}\right|^{2} \frac{P_{k 1,2}^{n, \mathrm{SP} 2(n)}}{\sigma^{2}+\sum_{l=1}^{L} J_{p l, 0}^{\mathrm{SP} 2(n)}}\right)\right\}
\end{aligned}
$$

where $\sigma^{2}$ denotes the Additive White Gaussian Noise(AWGN) variance. Here, it is assumed that all the channel gains are constant during two frames and the link between cooperative partners are symmetric, i.e., $h_{k 1, k 2}^{s s, n}=h_{k 2, k 1}^{s s, n}$ for all $k$. The factor $1 / 4$ in Equation (8) results from the fact that the transmission takes four slots in the cooperative scheme.

Let $P_{k 1, i}^{n, S P i(n)}+P_{k 2, i}^{n, S P i(n)}=P_{k i}^{n, S P i(n)}, i=1,2$ where $i$ denotes the $i$ th frame. This formula means that the average of the transmit power of the source node $P_{k 1, i}^{n, \operatorname{SP} i(n)}$ and that of the relay node $P_{k 2, i}^{n, \operatorname{SPi}(n)}$ is constrained to be $P_{k i}^{n, \operatorname{SP} i(n)}$, which is the allocated power on subcarrier $n$ at the source node for direct transmission. According to [42], the solution to this problem is the transmitted data rate and it is maximized when:

$$
\begin{gathered}
\left|h_{k 1, k 2}^{s s, n}\right|^{2} \frac{P_{k 1,1}^{n, \mathrm{SP} 1(n)}}{\sigma^{2}+\sum_{l=1}^{L} J_{p l, k 2}^{n}}=\left|h_{k 1,0}^{s s, n}\right|^{2} \frac{P_{k 1,1}^{n, \mathrm{SP} 1(n)}}{\sigma^{2}+\sum_{l=1}^{L} J_{p l, 0}^{n}}+\left|h_{k 2,0}^{s s, \mathrm{SP} 1(n)}\right|^{2} \frac{P_{k 2,1}^{n, \mathrm{SP} 1(n)}}{\sigma^{2}+\sum_{l=1}^{L} J_{p l, 0}^{\mathrm{SP} 1(n)}} \\
\left|h_{k 2, k 1}^{s s, n}\right|^{2} \frac{P_{k 2,2}^{n, \mathrm{SP} 2(n)}}{\sigma^{2}+\sum_{l=1}^{L} J_{p l, k 1}^{n}}=\left|h_{k 2,0}^{s s, n}\right|^{2} \frac{P_{k 2,2}^{n, \mathrm{SP} 2(n)}}{\sigma^{2}+\sum_{l=1}^{L} J_{p l, 0}^{n}}+\left|h_{k 1,0}^{s s, \operatorname{SP} 2(n)}\right|^{2} \frac{P_{k 1,2}^{n, \mathrm{SP} 2(n)}}{\sigma^{2}+\sum_{l=1}^{L} J_{p l, 0}^{\mathrm{SP} 2(n)}} \\
\text { for all } 1 \leq n \leq N, 1 \leq k \leq K
\end{gathered}
$$

Let:

$$
\gamma_{k 1, k 2}^{n}=\frac{\left|h_{k 1, k 2}^{s s, n}\right|^{2}}{\sigma^{2}+\sum_{l=1}^{L} J_{p l, k 2}^{n}}, \gamma_{k 1,0}^{n}=\frac{\left|h_{k 1,0}^{s s, n}\right|^{2}}{\sigma^{2}+\sum_{l=1}^{L} J_{p l, 0}^{n}}, \gamma_{k 2,0}^{n}=\frac{\left|h_{k 2,0}^{s s, n}\right|^{2}}{\sigma^{2}+\sum_{l=1}^{L} J_{p l, 0}^{n}}, \gamma_{k 2, k 1}^{n}=\frac{\left|h_{k 2, k 1}^{s s, n}\right|^{2}}{\sigma^{2}+\sum_{l=1}^{L} J_{p l, k 1}^{n}}
$$

Together with $P_{k 1, i}^{n, \operatorname{SP} i(n)}+P_{k 2, i}^{n, \operatorname{SP} i(n)}=P_{k, i}^{n, \operatorname{SP} i(n)}, i=1,2$ we can obtain that:

$$
\begin{aligned}
& P_{k 1,1}^{n, \operatorname{SP} 1(n)}=\frac{\gamma_{k 2,0}^{\mathrm{SP} 1(n)}}{\gamma_{k 1, k 2}^{n}-\gamma_{k 1,0}^{n}+\gamma_{k 2,0}^{\mathrm{SP} 1(n)}} P_{k 1}^{n, \mathrm{SP} 1(n)}, P_{k 2,1}^{n, \operatorname{SP} 1(n)}=\frac{\gamma_{k 1, k 2}^{n}-\gamma_{k 1,0}^{n}}{\gamma_{k 1, k 2}^{n}-\gamma_{k 1,0}^{n}+\gamma_{k 2,0}^{\mathrm{SP} 1(n)}} P_{k 1}^{n, \operatorname{SP} 1(n)} \\
& P_{k 1,2}^{n, \mathrm{SP} 2(n)}=\frac{\gamma_{k 2, k 1}^{n}-\gamma_{k 2,0}^{n}}{\gamma_{k 2, k 1}^{n}-\gamma_{k 2,0}^{n}+\gamma_{k 1,0}^{\mathrm{SP} 2(n)}} P_{k 2}^{n, \mathrm{SP} 2(n)}, P_{k 2,2}^{n, \operatorname{SP} 2(n)}=\frac{\gamma_{k 1,0}^{\mathrm{SP} 2(n)}}{\gamma_{k 2, k 1}^{n}-\gamma_{k 2,0}^{n}+\gamma_{k 1,0}^{\mathrm{SP} 2(n)}} P_{k 2}^{n, \mathrm{SP} 2(n)}
\end{aligned}
$$


Denote $\eta_{k 1}^{n, \operatorname{SP} 1(n)}, \eta_{k 2}^{n, \mathrm{SP} 2(n)}$ as the equivalent channel gain given by:

$$
\eta_{k 1}^{n, \mathrm{SP} 1(n)}=\frac{\gamma_{k 1, k 2}^{n} \gamma_{k 2,0}^{\mathrm{SP} 1(n)}}{\gamma_{k 1, k 2}^{n}-\gamma_{k 1,0}^{n}+\gamma_{k 2,0}^{\mathrm{SP} 1(n)}}, \eta_{k 2}^{n, \mathrm{SP} 2(n)}=\frac{\gamma_{k 2, k 1}^{n} \gamma_{k 1,0}^{\mathrm{SP} 2(n)}}{\gamma_{k 2, k 1}^{n}-\gamma_{k 2,0}^{n}+\gamma_{k 1,0}^{\mathrm{SP} 2(n)}}
$$

By now, we can unify the transmitted data rate as:

$$
\begin{aligned}
& I_{k 1}^{n, \mathrm{SP} 1(n)}\left(P_{k 1}^{n, \mathrm{SP} 1(n)}\right)=\frac{\Delta f}{4} \log _{2}\left\{1+\eta_{k 1}^{n, \operatorname{SP} 1(n)} P_{k 1}^{n, \operatorname{SP} 1(n)}\right\} \\
& I_{k 2}^{n, \mathrm{SP} 2(n)}\left(P_{k 2}^{n, \mathrm{SP} 2(n)}\right)=\frac{\Delta f}{4} \log _{2}\left\{1+\eta_{k 2}^{n, \operatorname{SP} 2(n)} P_{k 2}^{n, \operatorname{SP} 2(n)}\right\}
\end{aligned}
$$

The proportional fairness is used as the optimized objective to develop subcarrier-pair based resource allocation algorithm in order to maintain a balance between system efficiency and fairness. According to $[43,44]$ and the theorem in [45], we can readily deduce that there exists one unique proportionally fair allocation which can be attained by maximizing the objective function $\Psi=\sum_{k=1}^{K} \sum_{n=1}^{N} \omega_{k} \rho_{k n}\left(I_{k 1}^{n, \operatorname{SP1}(n)}+I_{k 2}^{n, \operatorname{SP2}(n)}\right)$ over the feasible set, where $\omega_{k}$ is the weighting factor to make the $K$ cooperative partners achieve the desirable transmitted data rate. Besides, we should keep the instantaneous interference introduced to the PUs below a certain threshold. The constraints include the aspects of satisfying the maximum power and interference constraints as well as the minimum rate requirements. Therefore, the resource allocation problem can be formulated mathematically as given in Equation (14). Constraint C1 corresponds to the subcarrier allocation constraint that each subcarrier $n$ only can be allocated to one cooperative partner. C2 and C3 define that the sum of all the transmission powers of a particular SU on different subcarriers can't be greater than the maximum allowed limit for that particular SU. C4 ensures the cumulative interference from all SUs and through all subcarriers on a particular PU should not be greater than the interference limit set. C5 ensure that each SU can obtain the minimum rate requirements. This constraint precludes the possibility of multiple SUs simultaneously transmitting at the same subcarrier:

$$
\begin{aligned}
& \max \quad \Psi=\sum_{k=1}^{K} \sum_{n=1}^{N} \omega_{k} \rho_{k n}\left(I_{k 1}^{n, \mathrm{SP} 1(n)}+I_{k 2}^{n, \mathrm{SP} 2(n)}\right) \\
& \text { subject to C1: } \sum_{k=1}^{K} \rho_{k n} \leq 1, \rho_{k n} \in\{0,1\}, \forall n \\
& \mathrm{C} 2: \sum_{n=1}^{N} \rho_{k n} P_{k 1}^{n, \mathrm{SP} 1(n)} \leq \frac{P_{t}}{2}, \mathrm{C} 3: \sum_{n=1}^{N} \rho_{k n} P_{k 2}^{n, \mathrm{SP} 2(n)} \leq \frac{P_{t}}{2} \\
& \mathrm{C} 4: \sum_{k=1}^{K} \sum_{n=1}^{N} \rho_{k n}\left[P_{k 1,1}^{n, \mathrm{SP} 1(n)} S_{k 1, p l}^{n}+P_{k 2,2}^{n, \mathrm{SP} 2(n)} S_{k 2, p l}^{n}+P_{k 1,2}^{n, \mathrm{SP} 2(n)} S_{k 1, p l}^{\mathrm{SP} 2(n)}+P_{k 2,1}^{n, \mathrm{SP} 1(n)} S_{k 2, p l}^{\mathrm{SP} 1(n)}\right] \leq I t h^{(l)} \\
& \mathrm{C} 5: \sum_{n=1}^{N} \rho_{k n}\left\{I_{k 1}^{n, \mathrm{SP} 1(n)}+I_{k 2}^{n, \mathrm{SP} 2(n)}\right\} \geq R_{k} \\
& \text { for all } l=\{1,2, \ldots, L\}, k=\{1,2, \ldots, K\}
\end{aligned}
$$

where $K$ denotes the number of cooperative partner, $k$ denotes the $k$ th cooperative partner, $L$ denotes the number of PUs, $l$ denotes the $l$ th PU, $\rho_{k n}$ is the allocation indicator that equals 1 when the $n$th subcarrier is allocated to the $k$ th cooperative partner and 0 otherwise, $P_{i}$ is the transmit power budget 
for each cooperative partner, the factor of $1 / 2$ for the terms of $P_{t} / 2$ which results from the fact that it is a normalization for the transmissions within the duration of a frame, It $h^{(l)}$ denotes the maximum allowable interference level at the $l$ th PU receiver, $R_{k}$ is the minimum transmitted data rate for $k$ th cooperative partners.

The optimal solution to Equation (14) can be found by performing an exhaustive search with computational complexity $O\left(K^{N} Z\right)$ [46], where $K^{N}$ is the number of possible subcarrier allocations and $Z$ is the complexity of a power allocation algorithm for each subcarrier allocation. To reduce the exponential computational complexity, a suboptimum resource allocation algorithm with less computational complexity is developed in the following. The dual decomposition approach is used to solve the problem. The dual problem of Equation (14) can be formulated as:

$$
\begin{aligned}
& \min _{\substack{\lambda^{(0)}, \lambda^{(1)}, \lambda^{(2)}, \lambda^{(3)}, \lambda^{(4)}}} \max _{\mathbf{P}, \mathbf{p}} \Psi=\sum_{k=1}^{K} \sum_{n=1}^{N} \omega_{k} \rho_{k n}\left(I_{k 1}^{n, \mathrm{SP} 1(n)}+I_{k 2}^{n, \mathrm{SP} 2(n)}\right)+\sum_{n=1}^{N} \lambda_{n}^{(0)}\left(1-\sum_{k=1}^{K} \rho_{k n}\right)+\sum_{k=1}^{K} \lambda_{k}^{(1)}\left\{\frac{P_{t}}{2}-\sum_{n=1}^{N} \rho_{k n} P_{k 1}^{n, \mathrm{~S}}\right. \\
& +\sum_{k=1}^{K} \lambda_{k}^{(2)}\left\{\frac{P_{t}}{2}-\sum_{n=1}^{N} \rho_{k n} P_{k 2}^{n, \mathrm{SP} 2(n)}\right\}+\sum_{l=1}^{L} \lambda_{l}^{(3)}\left\{I t h^{(l)}-\sum_{k=1}^{K} \sum_{n=1}^{N} \rho_{k n}\left[P_{k 1,1}^{n, \mathrm{SP} 1(n)} S_{k 1, p l}^{n}+P_{k 2,2}^{n, \mathrm{SP} 2(n)} S_{k 2, p l}^{n}\right.\right. \\
& \left.\left.+P_{k 1,2}^{n, \mathrm{SP} 2(n)} S_{k 1, p l}^{\mathrm{SP} 2(n)}+P_{k 2,1}^{n, \mathrm{SP} 1(n)} S_{k 2, p l}^{\mathrm{SP} 1(n)}\right]\right\}+\sum_{k=1}^{K} \lambda_{k}^{(4)}\left[\sum_{n=1}^{N} \rho_{k n}\left(I_{k 1}^{n, \mathrm{SP} 1(n)}+I_{k 2}^{n, \mathrm{SP} 2(n)}\right)-R_{k}\right] \\
& \text { subject to } P_{k i, j}^{n, \mathrm{SP} i(n)} \geq 0, \lambda=\left\{\lambda_{n}^{(0)}, \lambda_{k}^{(1)}, \lambda_{l}^{(2)}, \lambda_{k}^{(3)}, \lambda_{k}^{(4)}\right\} \geq 0 \\
& n \in\{1,2, \ldots, N\},\{i, j\} \in\{1,2\}, k \in\{1,2 \ldots, K\}
\end{aligned}
$$

where $i$ and $j$ denote the $i$ th and $j$ th SU of the $k$ th cooperative partner, respectively. The values of $\left\{\lambda_{n}^{(0)}, \lambda_{k}^{(1)}, \lambda_{k}^{(2)}, \lambda_{l}^{(3)}, \lambda_{k}^{(4)}\right\}$ are the introduced Lagrange multipliers. In the future using, we denote a vector shown in Equation (16):

$$
\begin{aligned}
& \lambda^{(0)}=\left[\lambda_{1}^{(0)}, \lambda_{2}^{(0)}, \cdots, \lambda_{N}^{(0)}\right]^{T}, \lambda^{(1)}=\left[\lambda_{1}^{(1)}, \lambda_{2}^{(1)}, \cdots, \lambda_{K}^{(1)}\right]^{T}, \lambda^{(2)}=\left[\lambda_{1}^{(1)}, \lambda_{2}^{(1)}, \cdots, \lambda_{K}^{(1)}\right]^{T} \\
& \lambda^{(3)}=\left[\lambda_{1}^{(2)}, \lambda_{2}^{(2)}, \cdots, \lambda_{L}^{(2)}\right]^{T}, \lambda^{(4)}=\left[\lambda_{1}^{(3)}, \lambda_{2}^{(3)}, \cdots, \lambda_{K}^{(3)}\right]^{T}
\end{aligned}
$$

The Equation (15) can be decomposed into two layers of sub-problems. In the lower layer, we can get $K$ sub-problems:

$$
\begin{aligned}
& \Phi^{k}\left(\boldsymbol{\lambda}^{(0)}, \boldsymbol{\lambda}^{(1)}, \boldsymbol{\lambda}^{(2)}, \boldsymbol{\lambda}^{(3)}, \boldsymbol{\lambda}^{(4)}\right)=\max _{\mathbf{P}, \boldsymbol{\rho}} \sum_{n=1}^{N} L_{k}^{n, \operatorname{SP} 1(n), \operatorname{SP} 2(n)} \rho_{k n} \\
& \text { subject to } \rho_{k n} \in\{0,1\}, \forall n \in\{1,2, \ldots, N\}, \forall k \in\{1,2, \ldots, K\} \\
& \boldsymbol{\rho}=\left\{\rho_{k n}\right\}, \mathbf{P}=\left\{P_{k 1,1}^{n, \mathrm{SP} 1(n)}, P_{k 1,2}^{n, \mathrm{SP} 2(n)}, P_{k 2,1}^{n, \mathrm{SP} 1(n)}, P_{k 2,2}^{n, \mathrm{SP} 2(n)}\right\} \geq 0 \\
& \text { where } \\
& L_{k}^{n, \operatorname{SP} 1(n), \operatorname{SP} 2(n)}\left(P_{k 1}^{n, \operatorname{SP1}(n)}, P_{k 2}^{n, \operatorname{SP} 2(n)}\right)=\omega_{k}\left(I_{k 1}^{n, \operatorname{SP} 1(n)}+I_{k 2}^{n, \operatorname{SP} 2(n)}\right)-\sum_{l=1}^{L} \lambda_{l}^{(3)}\left[P_{k 1,1}^{n, \operatorname{SP} 1(n)} S_{k 1, p l}^{n}+P_{k 2,2}^{n, \operatorname{SP} 2(n)} S_{k 2, p l}^{n}\right. \\
& \left.+P_{k 1,2}^{n, \mathrm{SP} 2(n)} S_{k 1, p l}^{\mathrm{SP} 2(n)}+P_{k 2,1}^{n, \mathrm{SP} 1(n)} S_{k 2, p l}^{\mathrm{SP} 1(n)}\right]-\lambda_{n}^{(0)}-\lambda_{k}^{(1)} P_{k 1}^{n, \operatorname{SP} 1(n)}-\lambda_{k}^{(2)} P_{k 2}^{n, \mathrm{SP} 2(n)}+\lambda_{k}^{(4)}\left(I_{k 1}^{n, \operatorname{SP} 1(n)}+I_{k 2}^{n, \mathrm{SP} 2(n)}\right) \\
& \forall n=1,2, \cdots, N, \forall k=1,2, \cdots, K
\end{aligned}
$$

We suppose that $\Gamma_{k}$ is the maximum value of the objective function in the lower layer. The master problem in the upper layer could be expressed as: 


$$
\mathrm{H}(\mathbf{P})=\min _{\substack{\lambda^{(0)}, \lambda^{(1)} \\ \lambda^{(2)}, \lambda^{(3)}, \lambda^{(4)}}} \sum_{k=1}^{K} \Gamma_{k}+\sum_{n=1}^{N} \lambda_{n}^{(0)}+\frac{1}{2} \sum_{k=1}^{K} \lambda_{k}^{(1)} P_{t}+\frac{1}{2} \sum_{k=1}^{K} \lambda_{k}^{(2)} P_{t}+\sum_{l=1}^{L} \lambda_{l}^{(3)} I t h^{(l)}-\sum_{k=1}^{K} \lambda_{k}^{(4)} R_{k}
$$

subject to $\left\{\lambda_{n}^{(0)}, \lambda_{k}^{(1)}, \lambda_{k}^{(2)}, \lambda_{l}^{(3)}, \lambda_{k}^{(4)}\right\} \geq 0$

Since a dual function is always optimized by first optimizing some variables and then optimizing the remaining ones. We define a subcarrier pairing parameter $\beta_{n, m} \in\{0,1\}$ that takes 1 if the $n$th subcarrier in $\mathrm{T} 1$ is pairing to $m$ th subcarrier in $\mathrm{T} 2$ and 0 otherwise. We first optimize the primal variables with the assumption that dual variables $\left\{\lambda_{n}^{(0)}, \lambda_{k}^{(1)}, \lambda_{k}^{(2)}, \lambda_{l}^{(3)}, \lambda_{k}^{(4)}\right\}$ are given. The resource allocation and subcarrier pairing process can be divided into four stages:

(a) Allocating the optimal power factor $\left\{P_{k 1,1}^{n, \operatorname{SP} 1(n)}, P_{k 1,2}^{n, \operatorname{SP} 2(n)}, P_{k 2,1}^{n, \operatorname{SP} 1(n)}, P_{k 2,2}^{n, \operatorname{SP} 2(n)}\right\}$ for SUs. $P_{k 1,1}^{n, \operatorname{SP} 1(n)}$ and $P_{k 2,2}^{n, \operatorname{SP} 2(n)}$ imply the power used for self-data transmission, respectively. $P_{k 1,2}^{n, \operatorname{SP} 2(n)}$ and $P_{k 2,1}^{n, \operatorname{SP} 1(n)}$ imply the power used for partner-data transmission, respectively.

4. Allocating the optimal set of subcarriers $\Omega_{k}$ for $k$ th cooperation partner, i.e., obtaining the optimal subcarrier allocation factor $\rho_{k n}$.

5. Optimal pairing process for the subcarriers which are allocated to $\Omega_{k}$, i.e., allocating the optimal subcarrier pairing factor $\beta_{n, m}$.

6. After the temporary optimal primal variables have been obtained in each iteration process, we would find the temporary optimal dual variables $\left\{\lambda_{n}^{(0)}, \lambda_{k}^{(1)}, \lambda_{k}^{(2)}, \lambda_{l}^{(3)}, \lambda_{k}^{(4)}\right\}$, which can minimize the objective function $\mathrm{H}(\mathbf{P})$ as shown in Equation (18).

\subsubsection{Power Allocation Algorithm}

Let $R_{k}^{n, \operatorname{SP1}(n), \operatorname{SP2}(n)}\left(P_{k 1}^{n, \operatorname{SP1}(n)}, P_{k 2}^{n, \operatorname{SP} 2(n)}\right)=I_{k 1}^{n, \operatorname{SP} 1(n)}+I_{k 2}^{n, \operatorname{SP} 2(n)}$, if we make $\rho_{k n}=1$, the power allocation can be determined in a water-filling fashion. Taking derivatives of $L_{k}^{n, \operatorname{SP} 1(n) \operatorname{SP} 2(n)}$ with respect to $P_{k 1}^{n, \mathrm{SP} 1(n)}, P_{k 2}^{n, \mathrm{SP} 2(n)}$.

$$
\begin{aligned}
& \frac{\partial L_{k}^{n, \operatorname{SP} 1(n), \operatorname{SP} 2(n)}\left(P_{k 1}^{n, \operatorname{SP} 1(n)}, P_{k 2}^{n, \operatorname{SP} 2(n)}\right)}{\partial P_{k 1}^{n, \operatorname{SP} 1(n)}}=\left(\omega_{k}+\lambda_{k}^{(4)}\right) \frac{\partial R_{k}^{n, \operatorname{SP} 1(n), \operatorname{SP} 2(n)}}{\partial P_{k 1}^{n, \operatorname{SP1}(n)}}-\lambda_{k}^{(1)}-\sum_{l=1}^{L} \lambda_{l}^{(3)}\left[A_{k 1,1}^{n, \operatorname{SP} 1(n)} S_{k 1, p l}^{n}+F_{k 2,1}^{n, \operatorname{SP} 1(n)} S_{k 2, p l}^{\operatorname{SP} 1(n)}\right]=0 \\
& \frac{\partial L_{k}^{n, \operatorname{SP} 1(n), \operatorname{SP} 2(n)}\left(P_{k 1}^{n, \operatorname{SP} 1(n)}, P_{k 2}^{n, \operatorname{SP} 2(n)}\right)}{\partial P_{k 2}^{n, \operatorname{SP} 2(n)}}=\left(\omega_{k}+\lambda_{k}^{(4)}\right) \frac{\partial R_{k}^{n, \operatorname{SP} 1(n), \operatorname{SP} 2(n)}}{\partial P_{k 2}^{n, \operatorname{SP} 2(n)}}-\lambda_{k}^{(2)}-\sum_{l=1}^{L} \lambda_{l}^{(3)}\left[D_{k 1,2}^{n, \operatorname{SP} 2(n)} S_{k 1, p l}^{\operatorname{SP} 2(n)}+B_{k 2,2}^{n, \operatorname{SP} 2(n)} S_{k 2, p l}^{n}\right]=0
\end{aligned}
$$

where

$$
\begin{aligned}
& A_{k 1,1}^{n, \mathrm{SP} 1(n)}=\frac{\gamma_{k 2,0}^{\mathrm{SP} 1(n)}}{\gamma_{k 1, k 2}^{n}-\gamma_{k 1,0}^{n}+\gamma_{k 2,0}^{\mathrm{SP} 1(n)}}, B_{k 2,2}^{n, \mathrm{SP} 2(n)}=\frac{\gamma_{k 1,0}^{\mathrm{SP} 2(n)}}{\gamma_{k 2, k 1}^{n}-\gamma_{k 2,0}^{n}+\gamma_{k 1,0}^{\mathrm{SP} 2(n)}} \\
& D_{k 1,2}^{n, \mathrm{SP} 2(n)}=\frac{\gamma_{k 2, k 1}^{n}-\gamma_{k 2,0}^{n}}{\gamma_{k 2, k 1}^{n}-\gamma_{k 2,0}^{n}+\gamma_{k 1,0}^{\mathrm{SP} 2(n)}}, F_{k 2,1}^{n, \mathrm{SP} 1(n)}=\frac{\gamma_{k 1, k 2}^{n}-\gamma_{k 1,0}^{n}}{\gamma_{k 1, k 2}^{n}-\gamma_{k 1,0}^{n}+\gamma_{k 2,0}^{\mathrm{SP} 1(n)}}
\end{aligned}
$$

Taking derivatives of $R_{k}^{n, \operatorname{SP} 1(n), \operatorname{SP} 2(n)}=I_{k 1}^{n, \operatorname{SP} 1(n)}+I_{k 2}^{n, \operatorname{SP} 2(n)}$ with respect to $P_{k 1}^{n, \operatorname{SP} 1(n)}, P_{k 2}^{n, \operatorname{SP} 2(n)}$, and according to Equation (19), we can get: 


$$
\begin{aligned}
& \frac{\partial R_{k}^{n, \operatorname{SP} 1(n), \operatorname{SP} 2(n)}}{\partial P_{k 1}^{n, \mathrm{SP} 1(n)}}=\frac{\Delta f}{4 \ln 2} \frac{\eta_{k 1}^{n, \mathrm{SP} 1(n)}}{1+\eta_{k 1}^{n, \operatorname{SP} 1(n)} P_{k 1}^{n, \operatorname{SP1}(n)}}=\frac{\Theta_{k, 1}^{n, \operatorname{SP} 1(n)}}{\omega_{k}+\lambda_{k}^{(4)}} \\
& \Theta_{k, 1}^{n, \operatorname{SP} 1(n)}=\lambda_{k}^{(1)}+\sum_{l=1}^{L} \lambda_{l}^{(3)}\left[A_{k 1,1}^{n, \operatorname{SP} 1(n)} S_{k 1, p l}^{n}+F_{k 2,1}^{n, \operatorname{SP} 1(n)} S_{k 2, p l}^{\operatorname{SP} 1(n)}\right] \\
& \frac{\partial R_{k}^{n, \mathrm{SP} 1(n), \mathrm{SP} 2(n)}}{\partial P_{k 2}^{n, \mathrm{SP} 2(n)}}=\frac{\Delta f}{4 \ln 2} \frac{\eta_{k 2}^{n, \mathrm{SP} 2(n)}}{1+\eta_{k 2}^{n, \mathrm{SP} 2(n)} P_{k 2}^{n, \mathrm{SP} 2(n)}}=\frac{\Theta_{k, 2}^{n, \mathrm{SP} 2(n)}}{\omega_{k}+\lambda_{k}^{(4)}} \\
& \Theta_{k, 2}^{n, \mathrm{SP} 2(n)}=\lambda_{k}^{(2)}+\sum_{l=1}^{L} \lambda_{l}^{(3)}\left[D_{k 1,2}^{n, \mathrm{SP} 2(n)} S_{k 1, p l}^{\mathrm{SP} 2(n)}+B_{k 2,2}^{n, \mathrm{SP} 2(n)} S_{k 2, p l}^{n}\right]
\end{aligned}
$$

Together with the constraint $P_{1}^{n, S P 1(n)}, P_{2}^{n, S P 2(n)} \geq 0$, the temporary optimal solution can be obtained:

$$
\begin{aligned}
& \frac{P_{k 1}^{n, \mathrm{SP} 1(n)}}{=}=\max \left\{0, \frac{\frac{\Delta f}{4 \ln 2} \eta_{k 1}^{n, \mathrm{SP} 1(n)}\left(\omega_{k}+\lambda_{k}^{(4)}\right)-\Theta_{k, 1}^{n, \mathrm{SP} 1(n)}}{\Theta_{k, 1}^{n, \mathrm{SP} 1(n)} \eta_{k 1}^{n, \operatorname{SP} 1(n)}}\right\} \\
& \frac{P_{k 2}^{n, \mathrm{SP} 2(n)}}{=} \max \left\{0, \frac{\frac{\Delta f}{4 \ln 2} \eta_{k 2}^{n, \mathrm{SP} 2(n)}\left(\omega_{k}+\lambda_{k}^{(4)}\right)-\Theta_{k, 2}^{n, \mathrm{SP} 2(n)}}{\Theta_{k, 2}^{n, \mathrm{SP} 2(n)} \eta_{k 2}^{n, \mathrm{SP} 2(n)}}\right\}
\end{aligned}
$$

The temporary SU's transmit power can be obtained for the given dual variables:

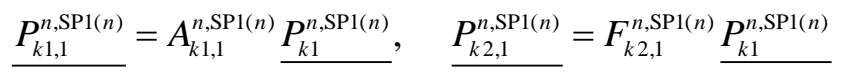

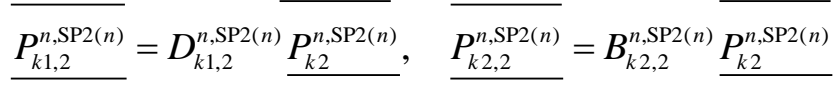

\subsubsection{Subcarrier Allocation Algorithm}

The subcarrier allocation constraint is that each subcarrier is allocated to no more than one SU cooperative partner, which prevents mutual interference among SUs. According to Section 3.1.1, we

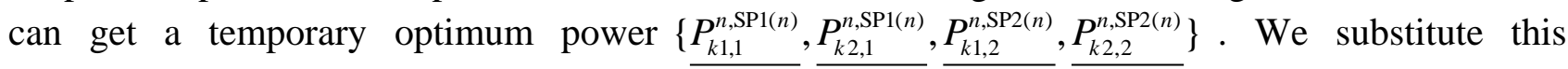
temporary optimum power vector into the objective function $L_{k}^{n, \operatorname{SP} 1(n), \operatorname{SP2}(n)}\left(P_{k 1}^{n, \operatorname{SP} 1(n)}, P_{k 2}^{n, \operatorname{SP} 2(n)}\right)$ and the objective function $R_{k}^{n, \operatorname{SP} 1(n), \operatorname{SP} 2(n)}\left(P_{k 1}^{n, \operatorname{SP} 1(n)}, P_{k 2}^{n, \operatorname{SP} 2(n)}\right)$ to obtain the temporary max value $L_{k}^{n, \operatorname{SP} 1(n), \operatorname{SP} 2(n)}$ and $R_{k}^{n, \operatorname{SP} 1(n), \operatorname{SP} 2(n)}$, respectively. Taking account of resource fairness, we can formulate the optimization problem of subcarrier allocation as:

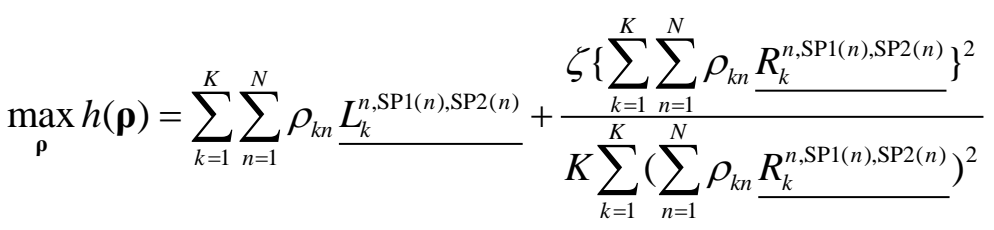

subject to $\rho_{k n} \in\{0,1\}, \sum_{k=1}^{K} \rho_{k n} \leq 1, \forall k, n$,

where $\boldsymbol{\rho}=\left\{\rho_{k n}\right\}, 1 \leq k \leq K, 1 \leq n \leq N$

where the value $\zeta$ is the weighting factor to balance the total transmitted data rate and fairness index among SUs. The bigger the value $\zeta$ is, the greater the fairness can be obtained, and otherwise the greater the transmitted data rate is. It is a mixed binary integer programming problem that is difficult to 
solve. To reduce the exponential computational complexity, a suboptimum subcarrier allocation algorithm with less computational complexity is developed. The pseudo-code of subcarrier allocation algorithm can be described as follows:

(1) Initialization

(i) Make $\rho_{k n}=0, \Omega_{k}=\varnothing, \forall k, n$

(2) For $n=1$ to $N$

(i) For $k=1$ to $K$

a) Make $\rho_{k n}=1$

b) Assign $V_{n k}=h(\boldsymbol{\rho})$ according to Equation (23)

c) Make $\rho_{k n}=0$.

(ii) Assign $k^{*}=\arg \max V_{n k}$

(iii) Assign $\rho_{k^{*} n}=1, \Omega_{k^{*}}=\Omega_{k^{*}} \cup\{n\}, \rho_{k n}=0, \forall k \neq k^{*}$

According to Section 3.1.2, we can obtain a temporary subcarrier allocation vector $\underline{\boldsymbol{\rho}}=\left\{\underline{\rho_{k n}}, 1 \leq k \leq K, 1 \leq n \leq N\right\}$ for the given dual variables.

\subsubsection{Subcarrier Pairing Algorithm}

The pairing constraint is that each subcarrier $\mathrm{m}$ in listening phase only pairs with at most one subcarrier $n$ in the relaying phase. We assume that the pairing for deferent frames is not the same. The pairing process of the subcarrier allocated to $k$ th cooperation partner can be expressed as:

$$
\begin{aligned}
& \text { Frame 1: } \max \sum_{n \in \Omega_{k}} \sum_{m \in \Omega_{k}} \beta_{n, m} I_{k 1}^{n, m} ; \text { Frame } 2: \max \sum_{n \in \Omega_{k}} \sum_{m^{\prime} \in \Omega_{k}} \beta_{n, m^{\prime}} I_{k 2}^{n, m^{\prime}} \\
& \text { subject to } \mathrm{C} 6: \sum_{m \in \Omega_{k}} \beta_{n, m} \leq 1, \mathrm{C} 7: \sum_{m^{\prime} \in \Omega_{k}} \beta_{n, m^{\prime}} \leq 1, \forall n \in \Omega_{k} \\
& \mathrm{C} 8: \sum_{n \in \Omega_{k}} \beta_{n, m} \leq 1, \mathrm{C} 9: \sum_{n \in \Omega_{k}} \beta_{n, m^{\prime}} \leq 1, \forall m \in \Omega_{k}, \forall m^{\prime} \in \Omega_{k} \\
& \mathrm{C} 10: \beta_{n, m} \in\{0,1\}, \mathrm{C} 11: \beta_{n, m^{\prime}} \in\{0,1\}
\end{aligned}
$$

Constraints C6, C8 and $\mathbf{C} 10$ correspond to the pairing constraint that each subcarrier $n$ in listening phase only pairs with one subcarrier $m$ in the relaying phase in the first frame. Constraint $\mathbf{C} 7, \mathrm{C} 9$ and $\mathbf{C} 11$ correspond to the pairing constraint that each subcarrier $n$ in listening phase only pairs with one subcarrier $m^{\prime}$ in the relaying phase for the second frame. We can obtain the temporary optimal $\underline{m}, \underline{m^{\prime}}$ for any $n$ as:

$$
\underline{m}=\underset{m}{\operatorname{argmax}}\left\{I_{k 1}^{n, m}\right\}, \underline{m^{\prime}}=\underset{m^{\prime}}{\operatorname{argmax}}\left\{I_{k 2}^{n, m^{\prime}}\right\}
$$

That is:

$$
\begin{aligned}
& \text { Frame 1: } \beta_{n, \underline{m}}=1, \operatorname{SP} 1(n)=\underline{m}, \beta_{n, m}=0 \quad \forall m \neq \underline{m} \\
& \text { Frame 2: } \beta_{n, \underline{m}^{\prime}}=1, \operatorname{SP} 2(n)=\underline{m^{\prime}}, \beta_{n, m^{\prime}}=0 \forall m^{\prime} \neq \underline{m^{\prime}}
\end{aligned}
$$


The subcarrier pairing scheme can be shown as following:

For $k=1$ to $K$

(a) $\Delta=\Omega_{k}, \Delta^{\prime}=\Omega_{k}, \beta_{n, m}=0, \beta_{n, m^{\prime}}=0, \forall n, m, m^{\prime} \in \Omega_{k}$

(b) While $\Delta \neq \varnothing, \Delta^{\prime} \neq \varnothing$

(i) $n \in \Delta, n^{\prime} \in \Delta^{\prime}$

(ii) Find $m \in \Delta, m^{\prime} \in \Delta^{\prime}$ satisfying $I_{k 1}^{n, m} \geq I_{k 1}^{n, l}, I_{k 2}^{n, m^{\prime}} \geq I_{k 2}^{n, l^{\prime}}, \forall l \in \Delta, \forall l^{\prime} \in \Delta^{\prime}$

(iii) Assign

$$
\begin{aligned}
& \operatorname{SP} 1(n)=m, \beta_{n, m}=1, \Delta=\Delta-\{n\}-\{m\} \\
& \operatorname{SP} 2\left(n^{\prime}\right)=m^{\prime}, \beta_{n^{\prime}, m^{\prime}}=1, \Delta^{\prime}=\Delta^{\prime}-\left\{n^{\prime}\right\}-\left\{m^{\prime}\right\}
\end{aligned}
$$

Through Section 3.1.3, we can obtain a temporary optimal subcarrier pairing vector $\underline{\beta_{n, m}}, \underline{\beta_{n, m^{\prime}}}$, $1 \leq\left\{n, m, m^{\prime}\right\} \leq N$ for the given dual variables.

\subsubsection{Optimizing the Dual Variables}

The optimal values of dual variables can be achieved iteratively by the sub-gradient method as follows:

$$
\begin{aligned}
& \lambda_{n}^{(0), i+1}=\left\{\lambda_{n}^{(0), i}-\alpha_{0, n}^{(i)}\left[1-\sum_{k=1}^{K} \rho_{k n}\right]\right\}^{+} \\
& \lambda_{k}^{(1), i+1}=\left\{\lambda_{k}^{(1), i}-\alpha_{1, k}^{(i)}\left[\frac{P_{t}}{2}-\sum_{n=1}^{N} \rho_{k n} P_{k 1}^{n, \operatorname{SP} 1(n)}\right]\right\}^{+} \\
& \lambda_{k}^{(2), i+1}=\left\{\lambda_{k}^{(2), i}-\alpha_{2, k}^{(i)}\left[\frac{P_{t}}{2}-\sum_{n=1}^{N} \rho_{k n} P_{k 2}^{n, \operatorname{SP} 2(n)}\right]\right\}^{+} \\
& \lambda_{l}^{(3), i+1}=\left\{\lambda_{l}^{(3), i}-\alpha_{3,,}^{(i)}\left[I t h^{(l)}-\sum_{k=1}^{K} \sum_{n=1}^{N} \rho_{k n}\left(P_{k 1,1}^{n, \operatorname{SP} 1(n)} S_{k 1, p l}^{n}+P_{k 2,2}^{n, \operatorname{SP} 2(n)} S_{k 2, p l}^{n}+P_{k 1,2}^{n, \operatorname{SP} 2(n)} S_{k 1, p l}^{\operatorname{SP} 2(n)}+P_{k 2,1}^{n, \operatorname{SP} 1(n)} S_{k 2, p l}^{\mathrm{SP}(n)}\right)\right]\right\}^{+} \\
& \lambda_{k}^{(4), i+1}=\left\{\lambda_{k}^{(4), i}-\alpha_{4, k}^{(i)}\left[\sum_{n=1}^{N} \rho_{k n}\left(I_{k 1}^{n, \operatorname{SP} 1(n)}+I_{k 2}^{n, \operatorname{SP} 2(n)}\right)-R_{k}\right]\right\}^{+}
\end{aligned}
$$

where $\left\{\alpha_{0, n}^{(i)}, \alpha_{l, k}^{(i)}, \alpha_{2, k}^{(i)}, \alpha_{3, l}^{(i)}, \alpha_{4, k}^{(i)}\right\}$ is a small positive step size for the $i$ th iteration, $i$ is the iteration number and $(x)^{+} \stackrel{\Delta}{=} \max (x, 0)$, with the appropriate step sizes, the iterations are convergent. The remaining issue is how to determine the step size $\left\{\alpha_{0, n}^{(i)}, \alpha_{1, k}^{(i)}, \alpha_{2, k}^{(i)}, \alpha_{3, l}^{(i)}, \alpha_{4, k}^{(i)}\right\}$. Clearly, performing a line search at each iteration process perform well. For a given current iteration $\lambda^{i}=\left\{\lambda_{n}^{(0), i}, \lambda_{k}^{(1), i}, \lambda_{k}^{(2), i}, \lambda_{l}^{(3), i}, \lambda_{k}^{(4), i}\right\}$ and a search direction $\mathbf{d}^{(i)}$, we compute step size $\boldsymbol{\alpha}^{k,(i)}$ by:

$$
\begin{aligned}
& \left\{\underline{\alpha_{0, n}^{(i)}}, \underline{\alpha_{l, k}^{(i)}}, \underline{\alpha_{2, k}^{(i)}}, \underline{\alpha_{3, l}^{(i)}}, \underline{\left.\alpha_{4, k}^{(i)}\right\}=\underset{\alpha_{0, n}^{(i)}, \alpha_{l, k}^{(i)}, \alpha_{2, k}^{(i)}, \alpha_{3, l}^{(i)}, \alpha_{4, k}^{(i)}}{\arg \min } Q\left\{\lambda_{n}^{(0), i}+\alpha_{0, n}^{(i)} d_{0}^{(i)}\right.}\right. \\
& \left., \lambda_{k}^{(1), i}+\alpha_{1, k}^{(i)} d_{1}^{(i)}, \lambda_{k}^{(2), i}+\alpha_{2, k}^{(i)} d_{2}^{(i)}, \lambda_{l}^{(3), i}+\alpha_{3, l}^{(i)} d_{3}^{(i)}, \lambda_{k}^{(4), i}-\alpha_{4, k}^{(i)} d_{4}^{(i)}\right\} \\
& \text { where } Q\left(\lambda_{n}^{(0)}, \lambda_{k}^{(1)}, \lambda_{k}^{(2)}, \lambda_{l}^{(3)}, \lambda_{k}^{(4)}\right)=\sum_{k=1}^{K} \Gamma_{k}+\sum_{n=1}^{N} \lambda_{n}^{(0)}+\sum_{k=1}^{K} \lambda_{k}^{(1)} P_{t} \\
& +\sum_{k=1}^{K} \lambda_{k}^{(2)} P_{t}+\sum_{l=1}^{L} \lambda_{l}^{(3)} I t h^{(l)}-\sum_{k=1}^{K} \lambda_{k}^{(4)} R_{k}, \quad \forall k, n
\end{aligned}
$$


Within each iteration process, the power allocation vectors can be updated respectively by Equation (22), the subcarrier allocation vectors can be updated respectively by subcarrier allocation algorithm shown in Section 3.1.2, the subcarrier pairing vectors can be updated respectively by subcarrier pairing algorithm which is shown in Section 3.1.3, with the updated value $\left\{\lambda_{n}^{(0), i}, \lambda_{k}^{(1), i}, \lambda_{k}^{(2), i}, \lambda_{l}^{(3), i}, \lambda_{k}^{(4), i}\right\}$. Therefore, the dual variable $\lambda^{i}$ will converge to the dual optimum $\stackrel{\lambda}{=}$ as $i \rightarrow \infty$ and the temporary primal optimum variable will also converge to the primal optimum value

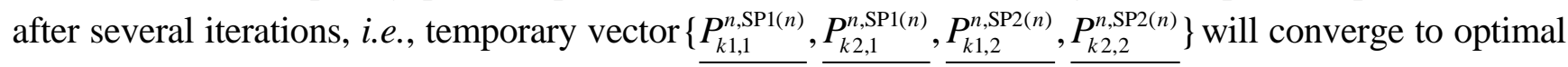

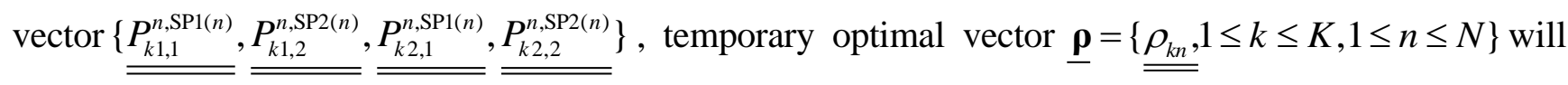
converge to the optimal vector $\underline{\underline{\boldsymbol{\rho}}}=\left\{\underline{\underline{\rho_{k n}}}, 1 \leq k \leq K, 1 \leq n \leq N\right\}$, temporary vector $\underline{\beta_{n, m}}, \underline{\beta_{n, m^{\prime}}}$ will converge to the optimal vector $\underline{\underline{\beta_{n, m}}}, \underline{\underline{\beta_{n, m^{\prime}}}}$.

\subsection{Resource Allocation with Partial CSI}

If full CSI can be achieved at the $k 1$ th and $k 2$ th $(1 \leq k \leq K)$ SU transmitter, the optimal subcarrier allocation vector $\stackrel{\rho}{=}$, subcarrier pairing vector $\underline{\underline{\beta_{n, m}}} \underline{\underline{\beta_{n, m^{\prime}}}}$, the power allocation vector

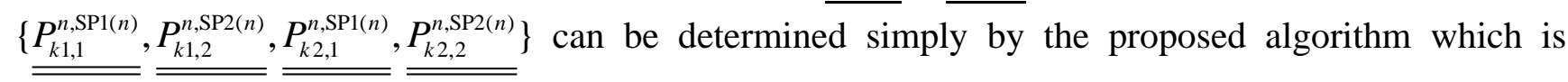
shown in Section 3.1. However, the practical case in which only partial CSI of the wireless channel between the secondary base station and SUs is available have to be considered. The CSI of non-adjacent link may be undesirable and even unavailable when the SUs are mobile. In this section, we investigate the optimal resource allocation in SU cooperation network with partial CSI at each transmitter. Specifically, the kith SU transmitter has full CSI of its adjacent links $h_{k i, k j}^{p s, n}(i \neq j), h_{k i, 0}^{p s, n}$ but only statistical CSI of non-adjacent $\operatorname{link} h_{k j, 0}^{p s, n}$. We assume that the SUs can know $h_{p l, k i}^{p s, n}, h_{p l, 0}^{p s, n}$, i.e., $h_{p l, k i}^{p s, n}, h_{p l, 0}^{p s, n}$ are still available at SU transmitter, and the link between cooperative users is symmetric for simplicity, i.e., $h_{k i, k j}^{p s, n}=h_{k j, k i}^{p s, n}$.

Under these assumptions and according to [47], the objective function:

$$
\Psi=\sum_{k=1}^{K} \sum_{n=1}^{N} \omega_{k} \rho_{k n}\left\{I_{k 1}^{n, \operatorname{SP} 1(n)}\left(P_{k 1}^{n, \operatorname{SP} 1(n)}\right)+I_{k 2}^{n, \operatorname{SP} 2(n)}\left(P_{k 2}^{n, \operatorname{SP} 2(n)}\right)\right\}
$$

can be rewritten as:

$$
\Psi=\sum_{k=1}^{K} \sum_{n=1}^{N} \omega_{k} \rho_{k n}\left\{\ln \overline{I_{k 1}^{n, \operatorname{SP} 1(n)}\left(P_{k 1}^{n, \mathrm{SP} 1(n)}\right)}+\ln \overline{I_{k 2}^{n, \operatorname{SP} 2(n)}\left(P_{k 2}^{n, \operatorname{SP} 2(n)}\right)}\right\}
$$

where $\overline{I_{k 1}^{n, \operatorname{SP} 1(n)}}$ and $\overline{I_{k 2}^{n, \operatorname{SP} 2(n)}}$ are defined as:

$$
\begin{aligned}
& \overline{I_{k 1}^{n, \operatorname{SP} 1(n)}\left(P_{1}^{n, \operatorname{SP1}(n)}\right)}=E_{h_{k 2,0}^{s, n}}\left[I_{k 1}^{n, \operatorname{SP} 1(n)}\left(P_{k 1}^{n, \operatorname{SP} 1(n)}\right)\right] \\
& \overline{I_{k 2}^{n, \operatorname{SP} 2(n)}\left(P_{2}^{n, \operatorname{SP} 2(n)}\right)}=E_{h_{k, 0}^{s, n}}\left[I_{k 2}^{n, \operatorname{SP} 2(n)}\left(P_{k 2}^{n, \operatorname{SP} 2(n)}\right)\right]
\end{aligned}
$$

In order to seek the optimal power allocation solution, we derive the explicit expressions for $\overline{I_{k 1}^{n, \mathrm{SP} 1(n)}}$ and $\overline{I_{k 2}^{n, \mathrm{SP} 2(n)}}$ described as: 
Let $h_{k i, 0}^{s s, n}=\left(\frac{1}{d_{k i, 0}}\right)^{v} x_{k i, 0}, i=1,2$, where $d_{k i, 0}$ denotes the distance between the $k$ ith SU and the AP, $v$ is the path-loss exponent, $x_{k i, 0},(i=1,2)$ is the normalized complex Gaussian random variable distributed as $C N(0,1)$, then at high signal-to-noise ratio (SNR):

$$
\begin{aligned}
& \overline{I_{k 1}^{n, \mathrm{SP} 1(n)}}=\frac{\Delta f}{4 \ln 2}\left\{\frac{P_{k 1}^{n, \mathrm{SP} 1(n)} \gamma_{k 1, k 2}^{n} W_{1}+W_{1}}{\gamma_{k 1, k 2}^{n}-\gamma_{k 1,0}^{n}}\left(1-\frac{P_{k 1}^{n, \mathrm{SP} 1(n)} \gamma_{k 1, k 2}^{n} W_{1}+W_{1}}{\gamma_{k 1, k 2}^{n}-\gamma_{k 1,0}^{n}}\right)-\frac{W_{1}}{\gamma_{k 1, k 2}^{n}-\gamma_{k 1,0}^{n}}\left(1-\frac{W_{1}}{\gamma_{k 1, k 2}^{n}-\gamma_{k 1,0}^{n}}\right)\right\} \\
& \overline{I_{k 2}^{n, \mathrm{SP} 2(n)}}=\frac{\Delta f}{4 \ln 2}\left\{\frac{P_{k 2}^{n, \mathrm{SP} 2(n)} \gamma_{k 2, k 1}^{n} W_{2}+W_{2}}{\gamma_{k 2, k 1}^{n}-\gamma_{k 2,0}^{n}}\left(1-\frac{P_{k 2}^{n, \mathrm{SP} 2(n)} \gamma_{k 2, k 1}^{n} W_{2}+W_{2}}{\gamma_{k 2, k 1}^{n}-\gamma_{k 2,0}^{n}}\right)-\frac{W_{2}}{\gamma_{k 2, k 1}^{n}-\gamma_{k 2,0}^{n}}\left(1-\frac{W_{2}}{\gamma_{k 2, k 1}^{n}-\gamma_{k 2,0}^{n}}\right)\right\}
\end{aligned}
$$

where $W_{1}, W_{2}$ in Equation (32) are defined as:

$$
W_{1}=\left[\left(\sigma^{2}+\sum_{l=1}^{L} J_{p l, 0}^{\mathrm{SP}(n)}\right) d_{k 2,0}^{2 v}\right]^{-1}, W_{2}=\left[\left(\sigma^{2}+\sum_{l=1}^{L} J_{p l, 0}^{\mathrm{SP} 1(n)}\right) d_{k 1,0}^{2 v}\right]^{-1}
$$

Proof: It is sufficient to show $\overline{I_{k 1}^{n, \mathrm{SP} 1(n)}}$, and $\overline{I_{k 2}^{n, \mathrm{SP} 2(n)}}$ can be derived in exactly the same way, then

$$
\begin{aligned}
& \overline{I_{k 1}^{n, \operatorname{SP} 1(n)}}=E_{h_{k 2,0}^{s s, n}}\left[I_{k 1}^{n, \operatorname{SP} 1(n)}\right]=E_{h_{k 2,0}^{s s, n}}\left\{\frac{\Delta f}{4} \log _{2}\left(1+\eta_{k 1}^{n, \operatorname{SP} 1(n)} P_{k 1}^{n, \operatorname{SP} 1(n)}\right)\right\} \\
& =E_{h_{k 2,0}^{s s, n}}\left\{\frac{\Delta f}{4} \log _{2}\left(1+\frac{\gamma_{k 1, k 2}^{n} \gamma_{k 2,0}^{\mathrm{SP} 1(n)}}{\gamma_{k 1, k 2}^{n}-\gamma_{k 1,0}^{n}+\gamma_{k 2,0}^{\mathrm{SP} 1(n)}} P_{k 1}^{n, \mathrm{SP} 1(n)}\right)\right\} \\
& =E_{h_{k 2,0}^{s s, n}}\left\{\frac{\Delta f}{4} \log _{2}\left(1+\frac{\gamma_{k 1, k 2}^{n} \frac{\left|h_{k 2,0}^{s s, \mathrm{SP} 1(n)}\right|^{2}}{\sigma^{2}+\sum_{l=1}^{L} J_{p l, 0}^{\mathrm{SP} 1(n)}}}{\gamma_{k 1, k 2}^{n}-\gamma_{k 1,0}^{n}+\frac{\left|h_{k 2,0}^{s s, \mathrm{SP} 1(n)}\right|^{2}}{\sigma^{2}+\sum_{l=1}^{L} J_{p l, 0}^{\mathrm{SP} 1(n)}}} P_{k 1}^{n, \mathrm{SP} 1(n)}\right)\right\} \\
& =\frac{\Delta f}{4} E_{h_{k 2,0}^{s s, n}}\left\{\log _{2}\left(1+\frac{\left.\gamma_{k 1, k 2}^{n}\left[\left(\sigma^{2}+\sum_{l=1}^{L} J_{p l, 0}^{n}\right) d_{k 2,0}^{2 v}\right]^{-1}\left|x_{k 2,0}\right|^{2} P_{k 1}^{n, \operatorname{SP} 1(n)}\right)}{\left(\gamma_{k 1, k 2}^{n}-\gamma_{k 1,0}^{n}+\frac{\left|x_{k 2,0}\right|^{2}}{\left(\sigma^{2}+\sum_{l=1}^{L} J_{p l, 0}^{\mathrm{SP} 1(n)}\right) d_{k 2,0}^{2 v}}\right)}\right\}\right.
\end{aligned}
$$

The third equality holds since we assume each transmission block is long enough to undergo different channel realizations as argued in [48]. Next, let:

$$
W_{1}=\left[\left(\sigma^{2}+\sum_{l=1}^{L} J_{p l, 0}^{\mathrm{SP} 1(n)}\right) d_{k 2,0}^{2 v}\right]^{-1}, y=\left|x_{k 2,0}\right|^{2}
$$

then according to [49], we can obtain: 


$$
\begin{aligned}
& \overline{I_{k 1}^{n, \mathrm{SP} 1(n)}}=E_{h_{k 2,0}^{s s, n}}\left[I_{k 1}^{n, \mathrm{SP} 1(n)}\right]=\frac{\Delta f}{4} E_{h_{k 2,0}^{s s, n}}\left\{\log _{2}\left[1+\frac{\gamma_{k 1, k 2}^{n} W_{1} y P_{k 1}^{n, \mathrm{SP} 1(n)}}{\gamma_{k 1, k 2}^{n}-\gamma_{k 1,0}^{n}+W_{1} y}\right]\right\} \\
& =\frac{\Delta f}{4} \int_{0}^{+\infty} \log _{2}\left[1+\frac{\gamma_{k 1, k 2}^{n} W_{1} y P_{k 1}^{n, \operatorname{SP} 1(n)}}{\gamma_{k 1, k 2}^{n}-\gamma_{k 1,0}^{n}+W_{1} y}\right] e^{-y} d y \\
& =\frac{\Delta f}{4 \ln 2} \int_{0}^{+\infty} \frac{e^{-y} P_{k 1}^{n, \operatorname{SP} 1(n)} \gamma_{k 1, k 2}^{n} W_{1}\left(\gamma_{k 1, k 2}^{n}-\gamma_{k 1,0}^{n}\right) d y}{\left(\gamma_{k 1, k 2}^{n}-\gamma_{k 1,0}^{n}+W_{1} y\right)\left(\gamma_{k 1, k 2}^{n}-\gamma_{k 1,0}^{n}+W_{1} y+P_{k 1}^{n, \operatorname{SP} 1(n)} \gamma_{k 1, k 2}^{n} W_{1} y\right)} \\
& =-\frac{\Delta f}{4 \ln 2} \int_{0}^{+\infty}\left\{\frac{e^{-y}}{y+\frac{\gamma_{k 1, k 2}^{n}-\gamma_{k 1,0}^{n}}{W_{1}}}-\frac{e^{-y}}{y+\frac{\gamma_{k 1, k 2}^{n}-\gamma_{k 1,0}^{n}}{P_{k 1}^{n, \mathrm{SP} 1(n)} \gamma_{k 1, k 2}^{n} W_{1}+W_{1}}}\right\} d y \\
& =-\frac{\Delta f}{4 \ln 2}\left\{-e^{\frac{\gamma_{k 1, k 2}^{n}-\gamma_{k 1,0}^{n}}{W_{1}}} \int_{-\infty}^{\frac{\gamma_{k 1,0}^{n}-\gamma_{k 1, k 2}^{n}}{W_{1}}} \frac{e^{y}}{y} d y+e^{\frac{\gamma_{k 1, k 2}^{n}-\gamma_{k 1,0}^{n}}{P_{k 1}^{n, S 1} 1(n)} \gamma_{k 1, k 2}^{n} W_{1}+W_{1}} \int_{-\infty}^{\frac{\gamma_{k 1,0}^{n}-\gamma_{k 1, k 2}^{n}}{P_{k 1}^{n, S P 1(n)} \gamma_{k 1, k 2}^{n} W_{1}+W_{1}}} \frac{e^{y}}{y} d y\right\} \\
& =\frac{-\Delta f}{4 \ln 2}\left\{-e^{\frac{\gamma_{k 1, k 2}^{n}-\gamma_{k 1,0}^{n}}{W_{1}}} \operatorname{Ei}\left(\frac{\gamma_{k 1,0}^{n}-\gamma_{k 1, k 2}^{n}}{W_{1}}\right)+e^{\frac{\gamma_{k 1, k 2}^{n}-\gamma_{k 1,0}^{n}}{P_{k 1}^{n, S \operatorname{Sl}(n)} \gamma_{k 1, k 2}^{n} W_{1}+W_{1}}} \operatorname{Ei}\left(\frac{\gamma_{k 1,0}^{n}-\gamma_{k 1, k 2}^{n}}{P_{k 1}^{n, S P 1(n)} \gamma_{k 1, k 2}^{n} W_{1}+W_{1}}\right)\right\}
\end{aligned}
$$

where $E i(\square)$ denotes the exponential integral function defined as $E i(t)=\int_{-\infty}^{t} e^{x} x^{-1} d x$ and $E_{i}(t)$ can be expanded asymptotically as:

$$
E i(t) \approx \frac{e^{t}}{t}\left(1+\sum_{i=1}^{+\infty} \frac{\prod_{k=1}^{i} k}{x^{i}}\right) \approx \frac{e^{t}}{t}\left[1+\frac{1}{t}+o\left(\frac{1}{t}\right)\right]
$$

Then using the assumption that SNR is high, we readily obtain Equation (32). This completes the proof.

We can use the resource allocation and subcarrier pairing algorithm which is proposed in Section 3.1 to solve the corresponding optimization Equation (14) once again. When partial CSI can be achieved by SUs, the optimal subcarrier allocation vector $\tilde{\rho}$, subcarrier pairing vector $\overbrace{\beta_{n, m}}, \overbrace{\beta_{n, m^{\prime}}}^{b}$ and optimal power allocation vector $\overbrace{P_{k 1}^{n, S P 1(n)}}, \overbrace{P_{k 2}^{n, S P 2(n)}}$ can be obtained. According to Equation (11), we can get

$$
\begin{aligned}
& \overbrace{P_{k 1,1}^{n, S P 1(n)}}=E_{h_{k 2,0}^{S S, n}}\{\frac{\gamma_{k 2,0}^{S P 1(n)}}{\gamma_{k 1, k 2}^{n}-\gamma_{k 1,0}^{n}+\gamma_{k 2,0}^{S P 1(n)}} \overbrace{P_{k 1}^{n, S P 1(n)}}^{W_{1}}\} \\
& =\int_{0}^{+\infty} \frac{W_{1} y}{\gamma_{k 1, k 2}^{n}-\gamma_{k 1,0}^{n}+W_{1} y} \overbrace{P_{k 1}^{n, S P 1(n)}}^{{ }^{-y}} d y \\
& =\overbrace{P_{k 1}^{n, S P 1(n)}} \int_{0}^{+\infty}\left\{1-\frac{\gamma_{k 1, k 2}^{n}-\gamma_{k 1,0}^{n}}{\gamma_{k 1, k 2}^{n}-\gamma_{k 1,0}^{n}+W_{1} y}\right\} e^{-y} d y \\
& =\overbrace{P_{k 1}^{n, S P 1(n)}}\left\{1+\frac{\left(\gamma_{k 1, k 2}^{n}-\gamma_{k 1,0}^{n}\right)}{W_{1}} e^{\frac{\left(\gamma_{k 1, k 2}^{n}-\gamma_{k 1,0}^{n}\right)}{W_{1}}}\right\} \int_{-\infty}^{\frac{\gamma_{k 1,0}^{n}-\gamma_{k 1, k 2}^{n}}{W_{1}}} \frac{e^{t}}{t} d t \\
& =\overbrace{P_{k 1}^{n, S P 1(n)}}+\frac{\overbrace{P_{k 1}^{n, S P 1(n)}}\left(\gamma_{k 1, k 2}^{n}-\gamma_{k 1,0}^{n}\right)}{W_{1}} e^{\frac{\left(\gamma_{k 1, k 2}^{n}-\gamma_{k 1,0}^{n}\right)}{W_{1}}} E i\left(\frac{\gamma_{k 1,0}^{n}-\gamma_{k 1, k 2}^{n}}{W_{1}}\right) \\
& =\frac{W_{1}}{\gamma_{k 1, k 2}^{n}-\gamma_{k 1,0}^{n}} \overbrace{P_{k 1}^{n, S P 1(n)}}
\end{aligned}
$$


Then we also can get:

$$
\begin{aligned}
& \overbrace{P_{k 2,1}^{n, S P 1(n)}}=\left(1-\frac{W_{1}}{\gamma_{k 1, k 2}^{n}-\gamma_{k 1,0}^{n}}\right) \overbrace{P_{k 1}^{n, S P 1(n)}}, \overbrace{P_{k 1,2}^{n, S P 2(n)}} \\
& =\left(1-\frac{W_{2}}{\gamma_{k 2, k 1}^{n}-\gamma_{k 2,0}^{n}}\right) \overbrace{P_{k 2}^{n, S P 2(n)}} \overbrace{P_{k 2,2}^{n, S P 2(n)}}=\frac{W_{2}}{\gamma_{k 2, k 1}^{n}-\gamma_{k 2,0}^{n}} \overbrace{P_{k 2}^{n, S P 2(n)}}
\end{aligned}
$$

Intuitively, the system performance would be degraded due to limited CSI though this scheme which does not require each cooperative node to have full CSI of nonadjacent link, as verified in the numerical simulation.

\subsection{Comparison with Classical Resource Allocation Algorithms}

Several existing schemes are compared with the proposed subcarrier-pair based resource allocation algorithm in terms of the system transmitted rate and fairness, respectively. These existing schemes include the following:

LWF-PI-without-SP: The subcarrier is allocated according to the channel gain. The messages transmitted on subcarrier $\mathrm{m}$ at the source node will be retransmitted on the same subcarrier $\mathrm{m}$ at the relay node. The power is allocated according to LWF-PI algorithm [23] on each subcarrier.

EPA-without-SP: The subcarrier is allocated according to the channel gain. The messages transmitted on subcarrier $\mathrm{m}$ at the source node will be retransmitted on the same subcarrier $\mathrm{m}$ at the relay node. The power is allocated equally on each subcarrier.

LWF-PI-with-SP: The subcarrier is allocated according to the channel gain. The messages transmitted on subcarrier $m$ at the source node will be retransmitted on subcarrier $n$, which is selected by proposed subcarrier pairing algorithm, at the relay node. The power is allocated according to LWF-PI algorithm [23] on each subcarrier.

EPA-with-SP: The subcarrier is allocated according to the channel gain. The messages transmitted on subcarrier $\mathrm{m}$ at the source node will be retransmitted on subcarrier $\mathrm{n}$, which is selected by proposed subcarrier pairing algorithm at the relay node. The power is allocated equally on each subcarrier.

Optimal-Scheme-with-SP: The subcarrier is allocated according to the channel gain. The messages transmitted on subcarrier $m$ at the source node will be retransmitted on subcarrier $n$, which is selected by proposed subcarrier pairing algorithm at the relay node. The power is allocated according to Optimal Scheme [37] on each subcarrier.

Partial CSI: the difference between the previous five algorithms and Partial CSI scheme is that the SU has only imperfect CSI of non-adjacent link under partial CSI scheme. In this case, the objective function is formulated by statistical methods.

\section{Simulation Results}

We have studied asymmetric or linear network with all SUs of $k$ th cooperative partner located in the same line. Specifically, $k 1$ th SU and the destination AP are fixed at $(0,0),(1,0)$ respectively, and $k 2$ th SU is located at $(d, 0), 0 \leq d \leq 1$, without loss of generality. The results for path loss exponent $v=2$ are presented and all channels are modeled as Rayleigh flat fading with AWGN. Some simulation parameters are shown in Table 3. 
Table 3. Simulation Parameters.

\begin{tabular}{cc}
\hline Parameter & Value \\
\hline Number of cooperative partners $K$ & 4 \\
Number of PUs $L$ & 2 \\
Number of subcarriers $N$ & 20 \\
Length of a slot $T s$ & $4 u \mathrm{~s}$ \\
value of amplitude $P P_{U}$ & $10 \times 10^{-3} \mathrm{~W}$ \\
$I t h^{(l)}(l=1,2)$ & $2.7 \times 10^{-3} \mathrm{~W}$ \\
average channel power gain & $10 \mathrm{~dB}$ \\
$\Delta f$ & $0.315 \mathrm{MHz}$ \\
$B_{2}$ & $1 \mathrm{MHz}$ \\
$B_{2}$ & $2 \mathrm{MHz}$ \\
\hline
\end{tabular}

Simulation results are presented in this section to verify the performance of the proposed subcarrier-pair based resource allocation algorithm. In our simulations, the CVX, a package for specifying and solving convex programs, is used to solve formulated optimization resource allocation problems.

The channel gains $h_{k i, k j}^{s s, n}, h_{k i, 0}^{s, n}, h_{k i, p l}^{s p, n}, h_{p l, k i}^{p s, n}, h_{p l, 0}^{p s, n}$ used in this paper are assumed to be Rayleigh fading, since the channel fading gains for different realizations of channel gain can be different, an average transmission capacity of 10,000 independent simulation runs is considered. And individual fairness index is defined as [46]:

$$
\text { fair }=\frac{\left\{\sum_{k=1}^{K} \sum_{n=1}^{N} \rho_{k n} \underline{\left.\underline{R_{k}^{n, \operatorname{SP} 1(n), \operatorname{SP} 2(n)}}\right\}^{2}}\right.}{K \sum_{k=1}^{K}\left(\sum_{n=1}^{N} \rho_{k n} \underline{\left.\underline{R_{k}^{n, \operatorname{SP} 1(n), \operatorname{SP} 2(n)}}\right)^{2}}\right.}
$$

The fairness ranges between 0 and 1 . The higher the value fairness is, the more fair the throughput distribution among SUs is.

\subsection{The System Transmitted Data Rate Obtained under Resource Allocation Algorithms}

As shown in Figure 4, the achievable maximum CR system transmitted data rate is plotted versus the power budget $P_{t}$. The upper curve denotes the transmitted data rate by proposed subcarrier-pair based resource allocation algorithm with full CSI. It can be noted that the proposed subcarrier-pair based resource allocation algorithm achieves the highest transmitted data rate under a given total power constraint. The transmitted data rate achieved using the proposed algorithm is the highest among that using LWF-PI-with-SP, Optimal-Scheme-with-SP algorithm, LWF-PI-without-SP algorithm and EPA algorithm. The main reason is that the proposed algorithms can make full use of the entire available interference threshold, while the LWF-PI algorithms can only guarantee that the total interference is under the interference threshold as shown in Figure 5. We observe that only the proposed algorithm outperforms the compared existing resource allocation algorithms. Therefore, we can conclude that the proposed subcarrier-pair based resource allocation algorithm makes valuable contribution to system transmitted data rate. 
Figure 4. Maximum transmitted data rate versus Power Budget.

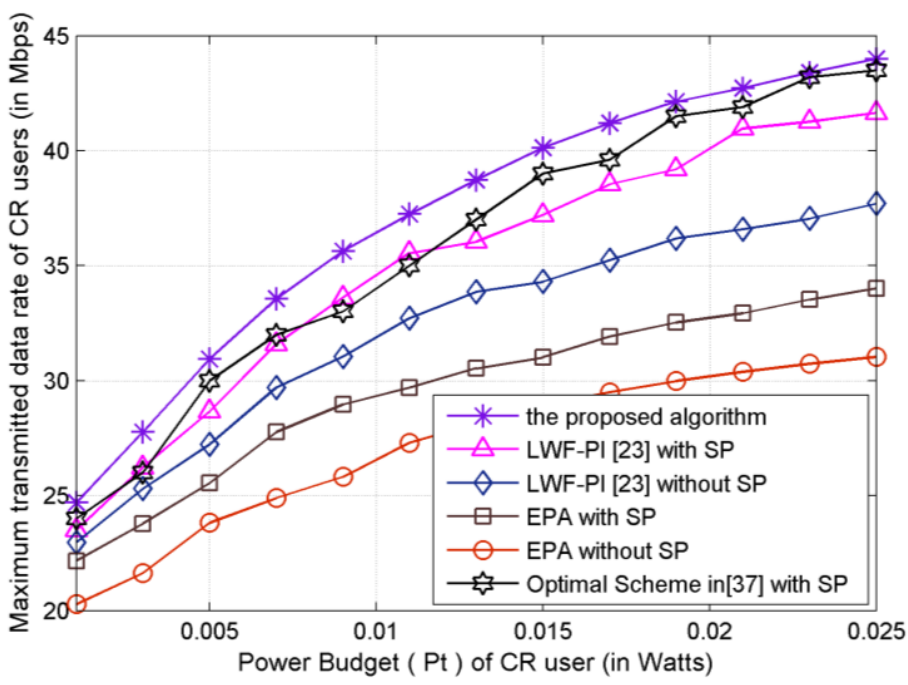

Figure 5. Power Budget versus interference introduced to PU bands.

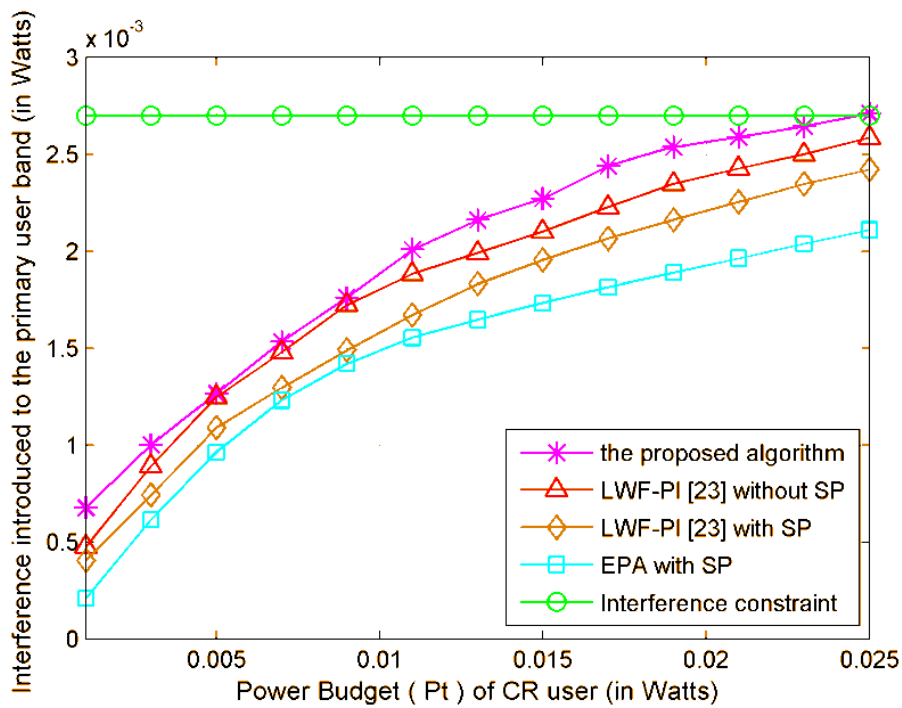

\subsection{The Fairness Index Obtained under Resource Allocation Algorithms}

Both the proposed resource allocation algorithm and EPA-with-SP algorithm exhibit the best fairness performance and LWF-PI-without-SP algorithm shows the least fairness as shown in Figure 6. Moreover, the fairness loss of EPA-with-SP allocation algorithm compared to the proposed subcarrier-pair based resource allocation algorithm is acceptable. In the LWF-PI-without-SP algorithm, LWF-PI-with-SP algorithm and Optimal-Scheme-with-SP algorithm, most power and subcarrier will be assigned to SUs with good channel conditions to improve system efficiency. Unlike the proposed subcarrier-pair based resource allocation algorithm and EPA schemes, these two schemes achieve significant performance improvement to ensure fairness among SUs. The fairness obtained by EPA-with-SP effectively approaches to 0.98 . Due to multiuser diversity, the fairness attained by the proposed algorithm is above 0.95 , while the achievable system transmitted data rate is higher than that of LWF-PI and EPA-without-SP algorithms. 
Figure 6. Power Budget versus Fairness index.

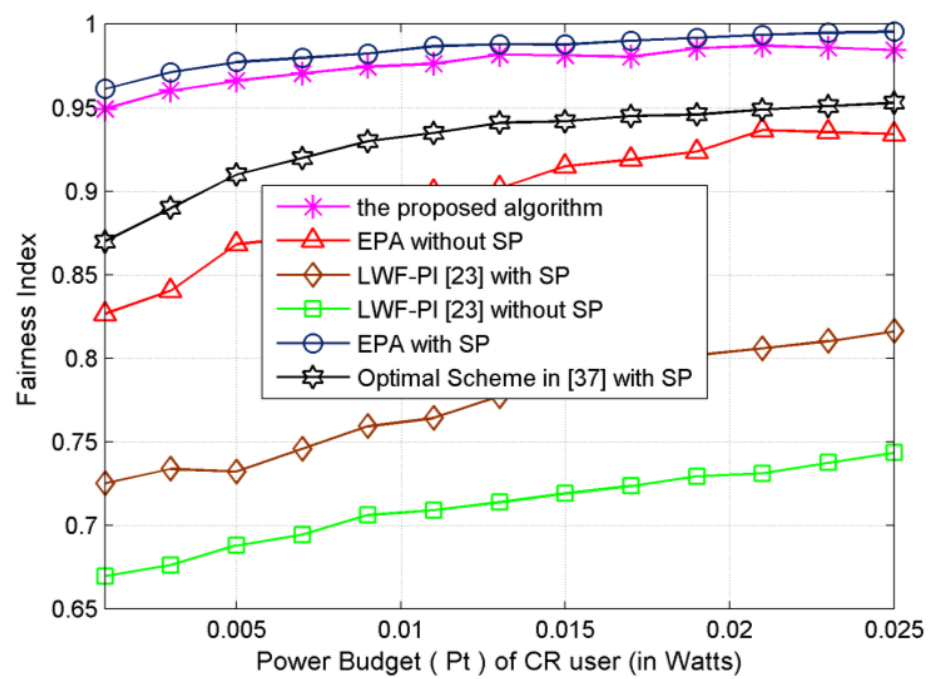

\subsection{The Transmitted Data Rate of Each SU for the Resource Allocation Schemes}

As shown in Figure 7, the achievable transmitted data rate of each SU is plotted. The transmitted data rate of each SU, under the proposed subcarrier-pair based resource allocation algorithm, is the most balanced and stable among EPA, LWF-PI and Optimal Scheme [37]. Especially under the LWF-PI algorithm and Optimal Scheme [37], some SUs with bad channel conditions have lower transmitted data rates, which would result in unsuccessful communication with destination node. We can conclude that the proposed subcarrier-pair based resource allocation algorithm makes valuable contribution to balance transmitted data rates among SUs.

Figure 7. Maximum transmitted data rate versus each SU (The power budget $P_{t}$ is $20 \times 10^{-3} \mathrm{~W}$ ).

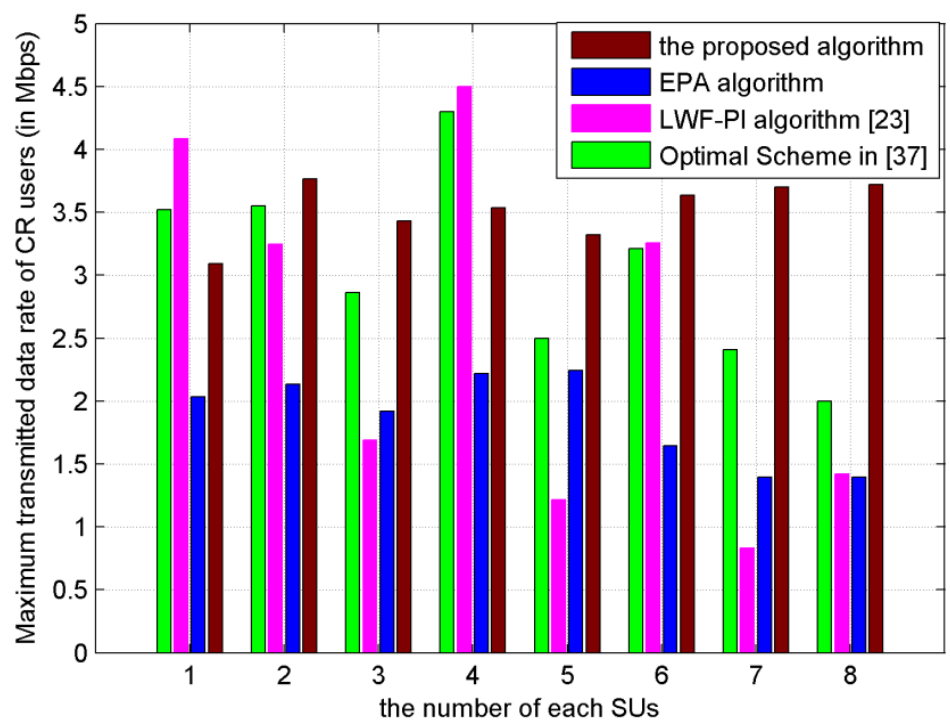

\subsection{The System Transmitted Data Rate Obtained under Full CSI and Partial CSI}

As shown in Figure 8, the performance of proposed subcarrier-pair based resource allocation algorithm is presented for the case that each SU has no CSI of non-adjacent link. It is shown that the 
system transmitted data rate decreases due to limited CSI compared to that of full CSI scenario. However, this scheme does not require the full CSI of non-adjacent link at each cooperative SU. Moreover, we show that the transmitted data rate of the proposed subcarrier-pair based resource allocation algorithm with partial CSI significantly outperforms that of EPA. The transmitted data rate is close to that of subcarrier-pair based resource allocation algorithm with full CSI.

Figure 8. Power Budget versus sum transmitted data rate.

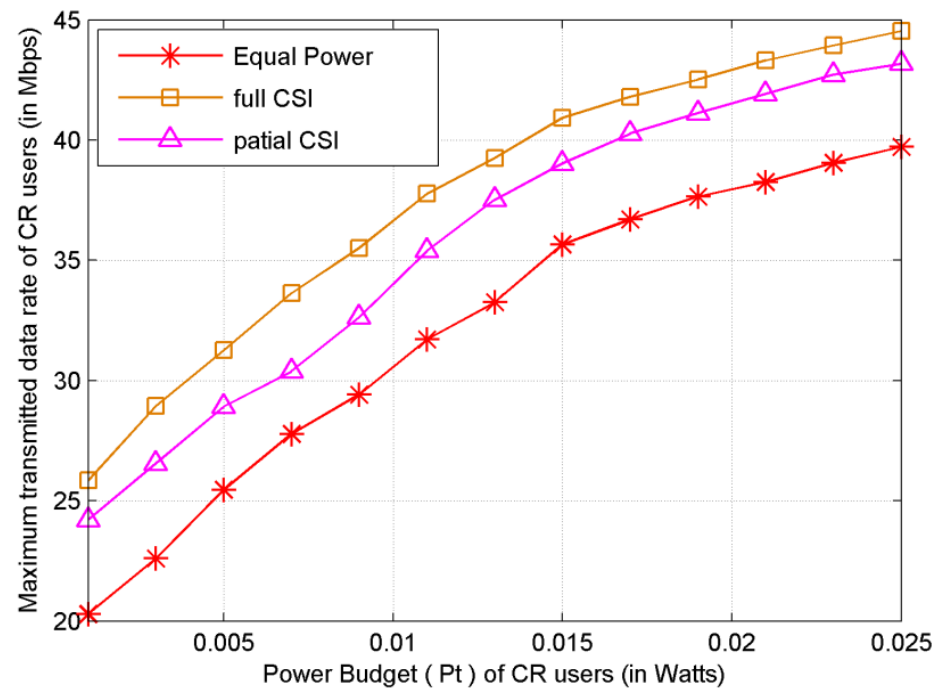

\subsection{The Transmitted Data Rate Obtained by SUs under Different Distance}

Last but not least, we have also studied how the distance between cooperative SUs impacts on the system performance. Under the proposed subcarrier-pair based resource allocation algorithm with proportional fairness, Figure 9 shows that the system has comparatively better performance when $d \in[0.4,0.6]$. This result provides a guideline for grouping and partner selection in user cooperative networks.

Figure 9. Maximum transmitted data rate versus distance between two cooperative SUs.

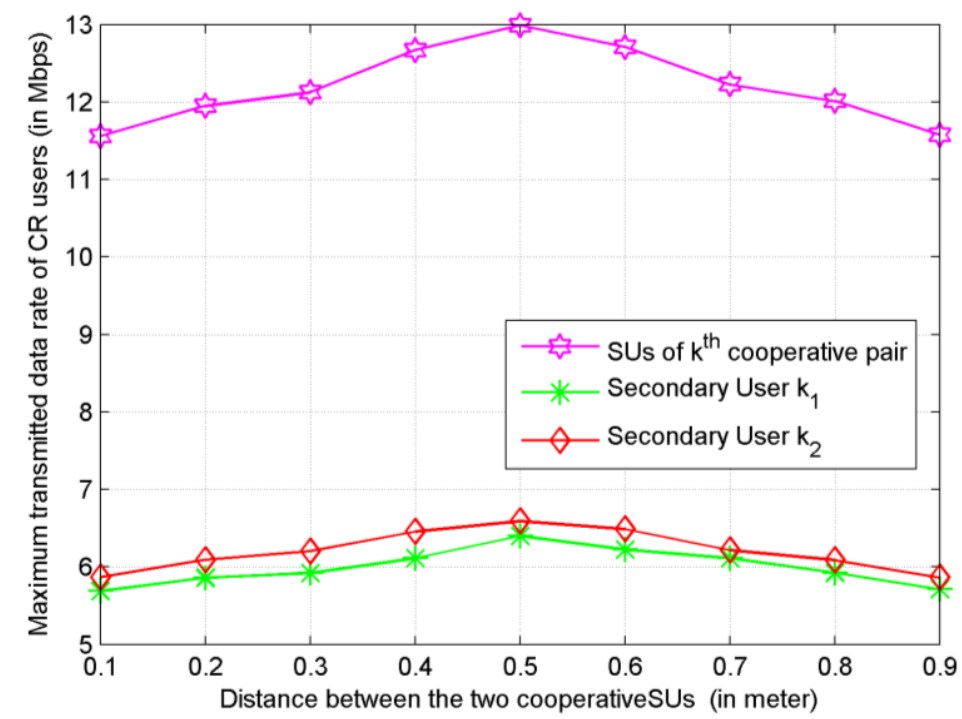




\section{Conclusions}

In this paper, we have developed a novel subcarrier-pair based resource allocation algorithm that maximizes the transmission data rate while the interference introduced to the PUs remains within a given limit. Using the proportional fairness as the optimized objective function, we can improve proportional fairness of resource allocation and achieve substantial transmitted data rate gains. The sum of power constraint for the source and relay nodes are considered. Moreover, we extended the analysis to the case that the CSI of nonadjacent link is not available at cooperative SUs, and found that even in this case the proposed schemes perform better than the classical schemes.

Compared to the existing resource allocation algorithms which are introduced in the paper, our algorithm considers that each secondary relay user has its own data to be transmitted. Simulation results have shown that, either in improving the system throughput or in improving fairness of resource allocation, the proposed subcarrier-pair based resource allocation algorithm offers the best performance conditions among several existing compared resource allocation algorithms under various power budgets, while keeping the interference introduced to PU bands below a specified threshold. Besides, the transmitted data rate of proposed subcarrier-pair based resource allocation algorithm obviously outperforms that of EPA scheme when only partial CSI can be obtained by SUs. The contribution by taking full advantage of the statistical non-adjacent links channel information is demonstrated clearly in the simulation results. In addition, we notice that the system efficiency loss of partial CSI scheme compared to that of full CSI scheme is acceptable.

\section{Acknowledgments}

This research was supported by the key project of Tianjin Key Technology R\&D Program (09ZCKFGX29200) of China.

\section{Conflict of Interest}

The authors declare no conflict of interest.

\section{References}

1. Simon, H. Cognitive radio brain-empowered wireless communications. IEEE J. Sel. Areas Commun. 2005, 23, 201-220.

2. Liang, Y.C.; Chen, K.C.; Li, G.Y. Cognitive radio networking and communications: An overview. IEEE Trans. Veh. Technol. 2011, 60, 3386-3407.

3. Laneman, J.N.; Tse, D.N.C.; Wornell, G.W. Cooperative diversity in wireless networks: Efficient protocols and outage behavior. IEEE Trans. Inf. Theory 2004, 50, 3062-3080.

4. Choi, Y.J.; Pak, W.; Xin, Y.; Rangarajan, S. Throughput analysis of cooperative spectrum sensing in Rayleigh-faded cognitive radio systems. IET Commun. 2012, 6, 1104-1110.

5. Kang, X.; Liang, Y.-C.; Nallanathan, A. Optimal power allocation for fading channels in cognitive radio networks: Ergodic capacity and outage capacity. IEEE Trans. Wirel. Commun. 2009, 8, 940-950. 
6. Kang, X.; Zhang, R.; Liang, Y.-C. Optimal power allocation strategies for fading cognitive radio channels with primary users outage constraint. IEEE J. Sel. Areas Commun. 2011, 29, 374-383.

7. Liu, J.; Shroff, N.B.; Sherali, H.D. Optimal power allocation in multi-relay MIMO cooperative networks: Theory and algorithms. IEEE J. Sel. Areas Commun. 2012, 30, 331-340.

8. Kwangyul, K.; Yoan, S. An Improved Power Allocation Scheme Using Particle Swarm Optimization in Cooperative Wireless Communication Systems. In Proceedings of the 17th Asia-Pacific Conference on Communications (APCC), Sabah, Malaysia, 2-5 October 2011; pp. 654-658.

9. Oberoi, J.S.; Pareek, U.; Naeem, M. EDA-Based Joint Power, Subcarrier Allocation and Relay Assignment Scheme for Multiuser Relaying in OFDMA-Based Cognitive Radio Systems. In Proceedings of the 2011 5th International Conference on Signal Processing and Communication Systems (ICSPCS), Honolulu, HI, USA, 12-14 December 2011; pp. 1-7.

10. Ding, Z.G.; Chin, W.H.; Leung, K.K. Distributed beamforming and power allocation for cooperative networks. IEEE Trans. Wirel. Commun. 2008, 7, 1817-1822.

11. Luo, J.; Blum, R.S.; Cimini, L.J. Decode-and-forward cooperative diversity with power allocation in wireless networks. IEEE Trans. Wirel. Commun. 2007, 6, 793-799.

12. Pan, Y.W.; Nix, A.; Beach, M. Distributed resource allocation for OFDMA-based relay networks. IEEE Trans. Veh. Technol. 2011, 60, 919-931.

13. Shen, Y.Y.; Feng, G.; Yang, B. Fair resource allocation and admission control in wireless multiuser amplify-and-forward relay networks. IEEE Trans. Veh. Technol. 2012, 61, 1383-1397.

14. Salem, M.; Adinoyi, A.; Rahman, M.; Yanikomeroglu, H.; Falconer, D.; Kim, Y.D. Fairness-aware radio resource management in downlink OFDMA cellular relay networks. IEEE Trans. Wirel. Commun. 2010, 9, 1628-1639.

15. Vu, H.N.; Kong, H.-Y. Joint subcarrier matching and power allocation in OFDM two-way relay systems. J. Commun. Netw. 2012, 14, 257-266.

16. Boostanimehr, H.; Bhargava, V.K. Selective subcarrier pairing and power allocation for DF OFDM relay systems with perfect and partial CSI. IEEE Trans. Wirel. Commun. 2011, 10, 4057-4067.

17. Dang, W.B.; Tao, M.X.; Mu, H. Subcarrier-pair based resource allocation for cooperative multi-relay OFDM systems. IEEE Trans. Wirel. Commun. 2010, 9, 1640-1649.

18. Sendonaris, A.; Erkip, E.; Aazhang, B. User cooperation diversity part I: System description. IEEE Trans. Commun. 2003, 51, 1927-1938.

19. Tourki, K.; Gesbert, D.; Deneire, L. Cooperative Diversity Using Per-User Power Control in the Multiuser MAC Channel. In Proceedings of the 2007 IEEE International Symposium on Information Theory, Nice, France, 24-29 June 2007; pp. 1911-1915.

20. Maciel, T.F.; Klein, A. On the performance, complexity, and fairness of suboptimal resource allocation for multiuser MIMO-OFDMA systems. IEEE Trans. Veh. Technol. 2010, 59, 406-419.

21. Papoutsis, V.D.; Fraimis, I.G.; Kotsopoulos, S.A. User selection and resource allocation algorithm with fairness in MISO-OFDMA. IEEE Commun. Lett. 2010, 14, 411-413.

22. Lu, W.S.; Ji, F.; Yu, H. A general resource allocation algorithm with fairness for SDMA/MISO/OFDMA systems. IEEE Commun. Lett. 2011, 15, 1072-1074. 
23. Wu, J.; Yang, L.X.; Liu, X. Subcarrier and Power allocation in OFDM Based Cognitive Radio Systems. In Proceedings of the 2011 Fourth International Conference on Intelligent Computation Technology and Automation (ICICTA), Shenzhen, China, 28-29 March 2011; pp. 728-731.

24. Mohanram, C.; Bhashyam, S. A sub-optimal joint subcarrier and power allocation algorithm for multiuser OFDM. IEEE Commun. Lett. 2005, 9, 685-687.

25. Tan, C.K.; Chuah, T.C.; Tan, S.W. Fair subcarrier and power allocation for multiuser orthogonal frequency-division multiple access cognitive radio networks using a Colonel Blotto game. IET Commun. 2011, 5, 1607-1618.

26. Mohamad, K.A.; Veluppillai, M.; Mehri, M. A dual-decomposition-based resource allocation for OFDMA networks with imperfect CSI. IEEE Trans. Veh. Technol. 2010, 59, 2394-2403.

27. Chen, C.-H.; Wang, C.-L.; Chen, C.-T. A resource allocation scheme for cooperative multiuser OFDM-based cognitive radio systems. IEEE Trans. Commun. 2011, 59, 3204-3215.

28. Yaacoub, E.; Dawy, Z. A survey on uplink resource allocation in OFDMA wireless networks. IEEE Commun. Surv. Tutor. 2012, 14, 322-337.

29. Nader, M.; Keivan, N.; Mohammad, G.K. Downlink radio resource allocation in OFDMA spectrum sharing environment with partial channel state information. IEEE Trans. Wirel. Commun. 2011, 10, 3482-3495.

30. Choi, K.W.; Hossain, E.; Kim, D.I. Downlink subchannel and power allocation in multi-cell OFDMA cognitive radio networks. IEEE Trans. Wirel. Commun. 2011, 10, 2259-2271.

31. Tianxiang, L.; Feifei, G.; Xian-Da, Z. Joint resource scheduling for relay-assisted broadband cognitive radio networks. IEEE Trans. Wirel. Commun. 2012, 11, 3090-4000.

32. Sabit, E.; Mohamed, M.A.; Khalid, A.Q. Random subcarrier allocation in OFDM-based cognitive radio networks. IEEE Trans. Signal Process. 2012, 60, 4758-4774.

33. Xu, H.; Li, B.C. Resource allocation with flexible channel cooperation in cognitive radio networks. IEEE Trans. Mob. Comput. 2013, 12, 957-970.

34. Bansal, G.; Hossain, M.D.J.; Bhargava, V.K. Subcarrier and power allocation for OFDMA-based cognitive radio systems with joint overlay and underlay spectrum access mechanism. IEEE Trans. Veh. Technol. 2013, 62, 1111-1122.

35. Naeem, M.; Pareek, U.; Lee, D.C.; Anpalagan, A. Estimation of distribution algorithm for resource allocation in green cooperative cognitive radio sensor networks. Sensors 2013, 13, 4884-4905.

36. Viswanath, P.; Tse, D.N.C.; Laroia, R. Opportunistic beamforming using dumb antennas. IEEE Trans. Inf. Theory 2002, 48, 1277-1294.

37. Bansal, G.; Hossain, M.D.J.; Bhargava, V.K. Optimal and suboptimal power allocation schemes for OFDM-based cognitive radio systems. IEEE Trans. Wirel. Commun. 2008, 7, 4710-4718.

38. Sami, M.A.; Gordon, L.S. Interference-aware radio resource allocation in OFDMA-based cognitive radio networks. IEEE Trans. Veh. Technol. 2011, 60, 1699-1713.

39. Kang, X.; Garg, H.K.; Liang, Y.-C. Optimal power allocation for OFDM-based cognitive radio with new primary transmission protection criteria. IEEE Trans. Wirel. Commun. 2010, 9, 2066-2075.

40. Liu, Y.; Chen, W. Limited-feedback-based adaptive power allocation and subcarrier pairing for OFDM DF relay. IEEE Trans. Veh. Technol. 2012, 61, 2559-2571. 
41. Sharma, S.; Shi, Y.; Liu, J. Network coding in cooperative communications: Friend or foe? IEEE Trans. Mob. Comput. 2012, 11, 1073-1085.

42. Zhang, Q.; Zhang, J.M.; Shao, C.J. Power Allocation for Regenerative Relay Channel with Rayleigh Fading. In Proceedings of IEEE 59th Vehicular Technology Conference (VTC 2004), Milan, Italy, 17-19 May 2004; pp. 1167-1171.

43. Le, L.B.; Hossain, E. Resource allocation for spectrum underlay in cognitive radio networks. IEEE Trans. Wirel. Commun. 2008, 7, 5306-5315.

44. Kelly, F.P.; Maulloo, A.K.; Tan, D.K.H. Rate control in communication networks: Shadow prices, proportional fairness and stability. J. Oper. Res. Soc. 1998, 49, 237-252.

45. Kim, H.; Keunyoung, K.; Youngnam, H. A Proportional Fair Scheduling for Multicarrier Transmission Systems. In Proceedings of IEEE 60th Vehicular Technology Conference (VTC2004-Fall), Los Angeles, CA, USA, 26-29 September 2004; pp. 409-413.

46. Shen, Z.; Andrews, J.G.; Evans, B.L. Adaptive resource allocation in multiuser OFDM systems with proportional rate constraints. IEEE Trans. Wirel. Commun. 2005, 4, 2726-2737.

47. Ma, Y.-T.; Lin, X.Q. Power Allocation with Proportional Fairness in User Cooperation Networks. In Proceedings of the 19th Annual Wireless and Optical Communications Conference (WOCC), Shanghai, China, 14-15 May 2010; pp. 1-5.

48. Host-Madsen, A.; Zhang, J.S. Capacity bounds and power allocation for wireless relay channels. IEEE Trans. Inf. Theory 2005, 51, 2020-2040.

49. Gradshteyn, I.S.; Ryzhik, I.M. Table of Integrals, Series, and Products, 7th ed.; Academic Press: San Diego, CA, USA, 2007.

(C) 2013 by the authors; licensee MDPI, Basel, Switzerland. This article is an open access article distributed under the terms and conditions of the Creative Commons Attribution license (http://creativecommons.org/licenses/by/3.0/). 